\title{
Total Syntheses of (-)-Spirooliganones A and B
}

\author{
Lin Wei, Mingxing Xiao and Zhixiang Xie* \\ State Key Laboratory of Applied Organic Chemistry \& College of Chemistry and Chemical \\ Engineering, Lanzhou University, Lanzhou 730000, P. R. China \\ E-mail: xiezx@1zu.edu.cn.
}

\section{Table of Contents}

1. General information

2. Experimental procedure

S2--S8

3. ${ }^{1} \mathrm{H}$ and ${ }^{13} \mathrm{C}$ NMR spectra of new compounds

S9--S31

General information: Oxygen- and moisture-sensitive reactions were carried out under argon atmosphere. Solvents were purified and dried by standard methods prior to use. All commercially available reagents were used without further purification unless otherwise noted. Column chromatography was performed on silica gel (200-300 mesh). NMR spectra were recorded on Bruker $400 \mathrm{MHz}$ and Oxford $600 \mathrm{MHz}$ spectrometers in the $\mathrm{CDCl}_{3}$ or acetone $\mathrm{d}_{6}$. Chemical shifts are reported as $\delta$ values relative to internal chloroform $\left(\delta 7.27\right.$ for $1 \mathrm{H} \mathrm{NMR}$ and 77.00 for ${ }^{13} \mathrm{C}$ NMR) and acetone- $\mathrm{d}_{6}\left(\delta 2.05\right.$ for ${ }^{1} \mathrm{H}$ NMR and 29.92 for ${ }^{13} \mathrm{C}$ NMR). High resolution mass spectra (HRMS) were obtained on a $4 \mathrm{G}$ mass spectrometer by using electrospray ionization (ESI) analyzed by quadrupole time-of-flight (QTof). Optical rotations were measured on a Rudolph Autoplo IV polarimeter. 


\section{Experimental procedure}

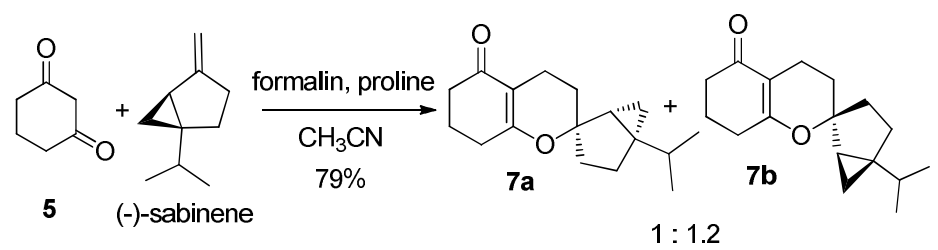

A mixture of (-)-sabinene $(75 \%, 29.15 \mathrm{~g}, 16.05 \mathrm{mmol}), 37 \%$ aqueous formalin $(15 \mathrm{~mL})$ and proline $(1.54 \mathrm{~g}, 1.34 \mathrm{mmol})$ in analysis of pure $\mathrm{CH}_{3} \mathrm{CN}(70 \mathrm{~mL})$ was heated at $95{ }^{\circ} \mathrm{C}$ and 1,3-cyclohexandione $(15.00 \mathrm{~g}, 13.38 \mathrm{mmol})$ was added during $6.5 \mathrm{~h}$. The mixture was heated another $5 \mathrm{~h}$, and then cooled to room temperature and diluted with water. The reaction mixture was extracted with ethyl acetate and the combined organic extracts were washed with brine, dried over $\mathrm{Na}_{2} \mathrm{SO}_{4}$ and concentrated in vacuo. Purification by column chromatography (petroleum ether/ethyl acetate $=6: 1)$ afforded the crude yellow solid $(27.6 \mathrm{~g}, 79 \%)$, then recrystallization of the crude solid from ethyl acetate/petroleum ether (1:2) afforded $\mathbf{7 b}(9.75 \mathrm{~g}, 28 \%)$ as a white solid.

7b: $\mathrm{Mp}: 90-92{ }^{\circ} \mathrm{C} ;[\alpha]^{20}{ }_{\mathrm{D}}-123.0(c 1.00, \mathrm{MeOH}){ }^{1} \mathrm{H}$ NMR $\left(400 \mathrm{MHz}, \mathrm{CDCl}_{3}\right) \delta$ 2.23-2.33 (m, $6 \mathrm{H}), 1.85-1.92(\mathrm{~m}, 2 \mathrm{H}), 1.74-1.80(\mathrm{~m}, 1 \mathrm{H}), 1.56-1.69(\mathrm{~m}, 4 \mathrm{H}), 1.37-1.45(\mathrm{~m}, 1 \mathrm{H}), 1.28(\mathrm{sept}, J=$ $6.8 \mathrm{~Hz}, 1 \mathrm{H}), 1.08(\mathrm{dd}, J=3.6 \mathrm{~Hz}, 8.0 \mathrm{~Hz}, 1 \mathrm{H}), 0.87(\mathrm{~d}, J=6.8 \mathrm{~Hz}, 3 \mathrm{H}), 0.83(\mathrm{~d}, J=6.8 \mathrm{~Hz}, 3 \mathrm{H})$, $0.77(\mathrm{dd}, J=3.6 \mathrm{~Hz}, 4.8 \mathrm{~Hz}, 1 \mathrm{H}), 0.39(\mathrm{dd}, J=5.2 \mathrm{~Hz}, 8.0 \mathrm{~Hz}, 1 \mathrm{H}) ;{ }^{13} \mathrm{C}$ NMR $\left(100 \mathrm{MHz}, \mathrm{CDCl}_{3}\right)$ $\delta 198.08,171.72,110.33,86.83,36.56,33.06,32.31,31.98,29.75,29.52,29.04,24.43,20.84$, 19.43, 19.36, 15.51, 11.53; HRMS (ESIMS) calculated for $\mathrm{C}_{17} \mathrm{H}_{25} \mathrm{O}_{2}[\mathrm{M}+\mathrm{H}]^{+} 261.1849$, found 261.1854
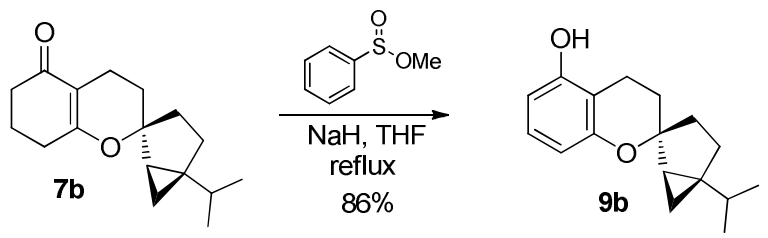

To a solution of $\mathrm{NaH}(70 \%, 0.90 \mathrm{~g}, 2.63 \mathrm{mmol})$ in THF $(100 \mathrm{~mL})$ at room temperature under Ar was slowly added $7 \mathbf{b}(5.69 \mathrm{~g}, 2.19 \mathrm{mmol})$ in THF $(15 \mathrm{~mL})$. The solution was stirred for $5 \mathrm{~min}$, followed by addition of Methyl benzenesulfinate $(3.42 \mathrm{~g}, 2.19 \mathrm{mmol})$. The mixture was heated to reflux for $4 \mathrm{~h}$, and then slowly warmed to room temperature. The reaction was quenched with a solution of $2 \mathrm{~N} \mathrm{HCl}(25 \mathrm{~mL})$ and extracted with ethyl acetate. The combined organic extracts were washed with water, brine, successively, dried over $\mathrm{Na}_{2} \mathrm{SO}_{4}$ and concentrated in vacuo. Purification by column chromatography (petroleum ether/ethyl acetate $=12: 1)$ afforded $9 \mathbf{b}(4.85 \mathrm{~g}, 86 \%)$ as a yellow liquid.

9b: $[\alpha]^{20}{ }_{\mathrm{D}}-52.0(c 1.00, \mathrm{MeOH}) ;{ }^{1} \mathrm{H}$ NMR $\left(400 \mathrm{MHz}, \mathrm{CDCl}_{3}\right) \delta 6.95(\mathrm{t}, J=8.4 \mathrm{~Hz}, 1 \mathrm{H}), 6.47$ $(\mathrm{d}, J=8.4 \mathrm{~Hz}, 1 \mathrm{H}), 6.34(\mathrm{~d}, J=8.0 \mathrm{~Hz}, 1 \mathrm{H}), 5.28-5.35(\mathrm{~m}, 1 \mathrm{H}), 2.70-2.85(\mathrm{~m}, 2 \mathrm{H}), 1.87-2.03(\mathrm{~m}$, $2 \mathrm{H}), 1.70-1.74(\mathrm{~m}, 3 \mathrm{H}), 1.52-1.59(\mathrm{~m}, 1 \mathrm{H}), 1.37$ (sept, 1H), $1.23(\mathrm{dd}, J=3.6 \mathrm{~Hz}, 8.0 \mathrm{~Hz}, 1 \mathrm{H})$, 0.91-0.98 (m, 7H), $0.45(\mathrm{dd}, J=5.2 \mathrm{~Hz}, 8.0 \mathrm{~Hz}, 1 \mathrm{H}) ;{ }^{13} \mathrm{C} \mathrm{NMR}\left(100 \mathrm{MHz}, \mathrm{CDCl}_{3}\right) \delta 156.11$, 153.92, 126.95, 109.72, 109.05, 106.09, 84.36, 32.98, 32.52, 31.96, 29.80, 29.46, 24.58, 19.58, 19.55, 16.89, 11.67; HRMS (ESIMS) calculated for $\mathrm{C}_{17} \mathrm{H}_{23} \mathrm{O}_{2}[\mathrm{M}+\mathrm{H}]^{+} 259.1693$, found 259.1690 . 


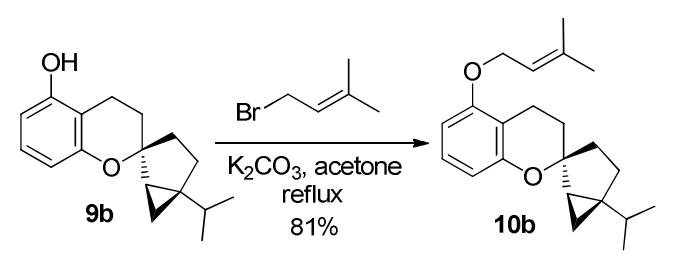

A mixture of $9 \mathbf{b}$ (160 mg, $0.62 \mathrm{mmol})$, prenyl bromide $(231 \mathrm{mg}, 1.55 \mathrm{mmol})$, and $\mathrm{K}_{2} \mathrm{CO}_{3}(342$ $\mathrm{mg}, 2.48 \mathrm{mmol})$ in anhydrous acetone $(8 \mathrm{~mL})$ was refluxed for $8.5 \mathrm{~h}$, then cooled to room temperature and diluted with water. The reaction mixture was extracted with ethyl acetate and the combined organic extracts were washed with brine, dried over $\mathrm{Na}_{2} \mathrm{SO}_{4}$ and concentrated in vacuo. Purification by column chromatography (petroleum ether/ethyl acetate $=50: 1)$ afforded 10b $(164$ $\mathrm{mg}, 81 \%)$ as a colorless liquid.

10b: $[\alpha]^{20}{ }_{\mathrm{D}}-90.0\left(c \mathrm{c} 0.10, \mathrm{CHCl}_{3}\right) ;{ }^{1} \mathrm{H}$ NMR $\left(400 \mathrm{MHz}, \mathrm{CDCl}_{3}\right) \delta 7.02(\mathrm{t}, J=8.0 \mathrm{~Hz}, 1 \mathrm{H}), 6.47$ $(\mathrm{d}, J=8.4 \mathrm{~Hz}, 1 \mathrm{H}), 6.40(\mathrm{~d}, J=8.4 \mathrm{~Hz}, 1 \mathrm{H}), 5.50(\mathrm{t}, J=6.0 \mathrm{~Hz}, 1 \mathrm{H}), 4,51(\mathrm{~d}, J=6.8 \mathrm{~Hz}, 2 \mathrm{H})$, 2.70-2.84 (m, 2H), 1.91-1.98 (m, 1H), 1.80-1.88 (m, 1H), $1.75(\mathrm{~s}, 3 \mathrm{H}), 1.72(\mathrm{~s}, 3 \mathrm{H}), 1.68-1.70(\mathrm{~m}$, $3 \mathrm{H}), 1.49-1.54(\mathrm{~m}, 1 \mathrm{H}), 1.35$ (sept, $J=6.8 \mathrm{~Hz}, 1 \mathrm{H}), 1.21(\mathrm{dd}, J=3.6 \mathrm{~Hz}, 8.0 \mathrm{~Hz}, 1 \mathrm{H}), 0.88-0.96$ $(\mathrm{m}, 7 \mathrm{H}), 0.42(\mathrm{dd}, J=5.2 \mathrm{~Hz}, 8.0 \mathrm{~Hz}, 1 \mathrm{H}),{ }^{13} \mathrm{C}$ NMR $\left(100 \mathrm{MHz}, \mathrm{CDCl}_{3}\right) \delta 157.20,155.98,137.12$, $126.63,120.27,110.91,109.97,102.39,84.10,65.00,32.99,32.59,32.24,30.01,29.50,25.79$, 24.70, 19.61, 19.57, 18.23, 17.29, 11.63; HRMS (ESIMS) calculated for $\mathrm{C}_{22} \mathrm{H}_{31} \mathrm{O}_{2}[\mathrm{M}+\mathrm{H}]^{+}$ 327.2319 , found 327.2320 .

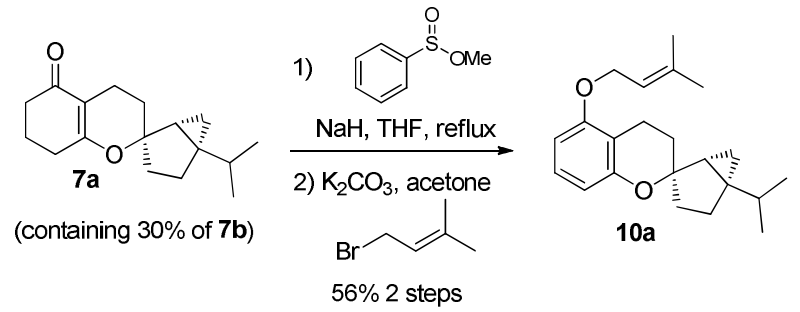

According to the identical procedures for $9 \mathbf{b}$ and $10 \mathbf{b}, 10 \mathbf{a}$ could be prepared and purified by column chromatography in $56 \%$ overall yield from $7 \mathbf{a}$.

10a: $[\alpha]^{21}{ }_{\mathrm{D}}-5.0\left(c\right.$ 1.00, $\left.\mathrm{CHCl}_{3}\right) ;{ }^{1} \mathrm{H}$ NMR $\left(400 \mathrm{MHz}, \mathrm{CDCl}_{3}\right) \delta 7.08(\mathrm{t}, J=8.0 \mathrm{~Hz}, 1 \mathrm{H}), 6.52$ $(\mathrm{d}, J=8.4 \mathrm{~Hz}, 1 \mathrm{H}), 6.45(\mathrm{~d}, J=8.0 \mathrm{~Hz}, 1 \mathrm{H}), 5.57(\mathrm{~s}, 1 \mathrm{H}), 4.57(\mathrm{~d}, J=6.4 \mathrm{~Hz}, 2 \mathrm{H}), 2.80-2.87(\mathrm{~m}$, $1 \mathrm{H}), 2.68-2.76(\mathrm{~m}, 1 \mathrm{H}), 2.06(\mathrm{t}, J=10.4 \mathrm{~Hz}, 1 \mathrm{H}), 1.91-1.93(\mathrm{~m}, 2 \mathrm{H}), 1.81-1.89(\mathrm{~m}, 7 \mathrm{H}), 1.66(\mathrm{dd}$, $J=8.0 \mathrm{~Hz}, 12.0 \mathrm{~Hz}, 1 \mathrm{H}), 1.43-1.50(\mathrm{~m}, 1 \mathrm{H}), 1.26-1.33(\mathrm{~m}, 2 \mathrm{H}), 1.06(\mathrm{~d}, J=6.8 \mathrm{~Hz}, 3 \mathrm{H}), 0.99(\mathrm{~d}$, $J=6.8 \mathrm{~Hz}, 3 \mathrm{H}), 0.47(\mathrm{dd}, J=5.6 \mathrm{~Hz}, 7.6 \mathrm{~Hz}, 1 \mathrm{H}), 0.35(\mathrm{t}, J=4.4 \mathrm{~Hz}, 1 \mathrm{H}) ;{ }^{13} \mathrm{C} \mathrm{NMR}(100 \mathrm{MHz}$, $\left.\mathrm{CDCl}_{3}\right) \delta 157.1,154.9,136.8,126.6,120.4,111.3,110.1,102.3,64.9,34.2,32.9,32.4,30.1,28.2$, 25.7, 25.4, 20.0, 19.7, 18.4, 18.2, 12.4; HRMS (ESIMS) calculated for $\mathrm{C}_{22} \mathrm{H}_{31} \mathrm{O}_{2}[\mathrm{M}+\mathrm{H}]^{+}$ 327.2319, found 327.2326.

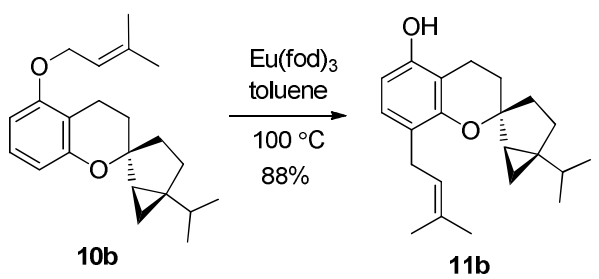


To a solution of $\mathbf{1 0 b}(110 \mathrm{mg}, 0.34 \mathrm{mmol})$ in toluene $(6 \mathrm{~mL}), \mathrm{Eu}(\mathrm{fod})_{3}(70 \mathrm{mg}, 0.067 \mathrm{mmol})$ was added, and the solution was heated at $100{ }^{\circ} \mathrm{C}$ until TLC analysis evidenced the complete conversion $(9.5 \mathrm{~h})$. The reaction was worked up by evaporation. Purification by column chromatography (petroleum ether/ethyl acetate $=10: 1)$ afforded $\mathbf{1 1 b}(97 \mathrm{mg}, 88 \%)$ as a canary yellow liquid.

11b: $[\alpha]^{20}{ }_{\mathrm{D}}-70.0(c 0.10, \mathrm{MeOH}) ;{ }^{1} \mathrm{H} \mathrm{NMR}\left(400 \mathrm{MHz}, \mathrm{CDCl}_{3}\right) \delta 6.83(\mathrm{~d}, J=8.0 \mathrm{~Hz}, 1 \mathrm{H}), 6.28$ $(\mathrm{d}, J=8.4 \mathrm{~Hz}, 1 \mathrm{H}), 5.33(\mathrm{t}, J=7.2 \mathrm{~Hz}, 1 \mathrm{H}), 4.82(\mathrm{~d}, J=6.4 \mathrm{~Hz}, 1 \mathrm{H}), 3.19-3.29(\mathrm{~m}, 2 \mathrm{H}), 2.72-2.86$ $(\mathrm{m}, 2 \mathrm{H}), 1.97-2.04(\mathrm{~m}, 1 \mathrm{H}), 1.86-1.94(\mathrm{~m}, 1 \mathrm{H}), 1.71-1.75(\mathrm{~m}, 9 \mathrm{H}), 1.58-1.64(\mathrm{~m}, 1 \mathrm{H}), 1.37$ (sept, $J$ $=6.8 \mathrm{~Hz}, 1 \mathrm{H}), 1.23(\mathrm{dd}, J=3.6,8.0 \mathrm{~Hz}, 1 \mathrm{H}), 0.97(\mathrm{~d}, J=6.8 \mathrm{~Hz}, 3 \mathrm{H}), 0.92(\mathrm{~d}, J=6.8 \mathrm{~Hz}, 3 \mathrm{H})$, $0.88(\mathrm{t}, J=4.0 \mathrm{~Hz}, 1 \mathrm{H}), 0.42(\mathrm{dd}, J=5.2 \mathrm{~Hz}, 8.0 \mathrm{~Hz}, 1 \mathrm{H}) ;{ }^{13} \mathrm{C} \mathrm{NMR}\left(100 \mathrm{MHz}, \mathrm{CDCl}_{3}\right) \delta 153.6$, 151.9, 131.3, 126.6, 123.5, 122.1, 108.7, 105.3, 84.0, 33.3, 32.8, 32.6, 29.8, 29.2, 28.1, 25.8, 24.7, 19.6, 17.7, 17.2, 11.7; HRMS (ESIMS) calculated for $\mathrm{C}_{22} \mathrm{H}_{31} \mathrm{O}_{2}[\mathrm{M}+\mathrm{H}]^{+} 327.2319$, found 327.2316 .

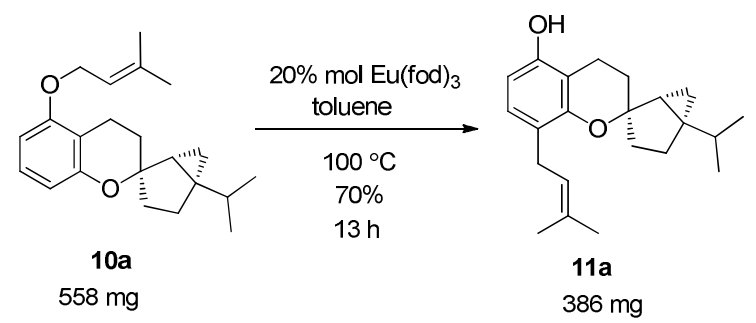

11a: $[\alpha]^{20}{ }_{\mathrm{D}}-35.0\left(c\right.$ 1.00, MeOH); ${ }^{1} \mathrm{H}$ NMR $\left(400 \mathrm{MHz}, \mathrm{CDCl}_{3}\right) \delta 6.84(\mathrm{~d}, J=8.0 \mathrm{~Hz}, 1 \mathrm{H}), 6.29$ $(\mathrm{d}, J=8.0 \mathrm{~Hz}, 1 \mathrm{H}), 5.36(\mathrm{t}, J=6.8 \mathrm{~Hz}, 1 \mathrm{H}), 4.80-4.83(\mathrm{~m}, 1 \mathrm{H}), 3.19-3.32(\mathrm{~m}, 2 \mathrm{H}), 2.75-2.82(\mathrm{~m}$, $1 \mathrm{H}), 2.60-2.67(\mathrm{~m}, 1 \mathrm{H}), 1.98-2.08(\mathrm{~m}, 1 \mathrm{H}), 1.86-1.96(\mathrm{~m}, 2 \mathrm{H}), 1.73-1.82(\mathrm{~m}, 7 \mathrm{H}), 1.64(\mathrm{dd}, J=$ $4.0 \mathrm{~Hz}, 8.0 \mathrm{~Hz}, 1 \mathrm{H}), 1.43$ (sept, $J=6.8 \mathrm{~Hz}, 1 \mathrm{H}), 1.22-1.30(\mathrm{~m}, 2 \mathrm{H}), 1.05$ (d, $J=6.8 \mathrm{~Hz}, 3 \mathrm{H}), 0.97$ $(\mathrm{d}, J=6.8 \mathrm{~Hz}, 3 \mathrm{H}), 0.46(\mathrm{dd}, J=5.6 \mathrm{~Hz}, 8.0 \mathrm{~Hz}, 1 \mathrm{H}), 0.35(\mathrm{t}, J=4.4 \mathrm{~Hz}, 1 \mathrm{H}) ;{ }^{13} \mathrm{C} \mathrm{NMR}(100$ $\left.\mathrm{MHz}_{\mathrm{CDCl}}\right) \delta 152.3,151.8,131.5,126.5,123.4,122.1,108.9,105.2,86.3,34.3,32.7,32.5,30.5$, 28.0, 27.7, 25.7, 25.4, 20.1, 19.7, 18.3, 17.7, 12.6; HRMS (ESIMS) calculated for $\mathrm{C}_{22} \mathrm{H}_{31} \mathrm{O}_{2}[\mathrm{M}+$ $\mathrm{H}]^{+} 327.2319$, found 327.2316 .
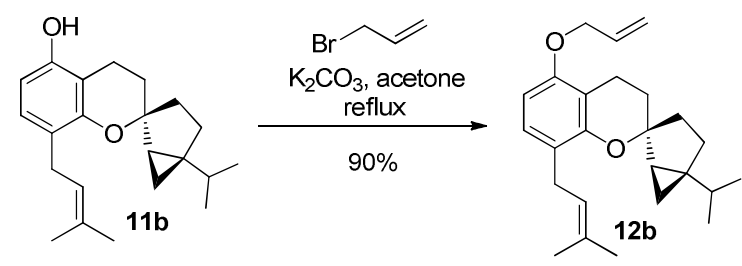

A mixture of 11b (594 mg, $1.82 \mathrm{mmol})$, Allyl bromide (880 mg, $7.28 \mathrm{mmol})$, and $\mathrm{K}_{2} \mathrm{CO}_{3}(1.51 \mathrm{~g}$, $10.92 \mathrm{mmol})$ in anhydrous acetone $(25 \mathrm{~mL})$ was refluxed for $18 \mathrm{~h}$, then cooled to room temperature and diluted with water. The reaction mixture was extracted with ethyl acetate and the combined organic extracts were washed with brine, dried over $\mathrm{Na}_{2} \mathrm{SO}_{4}$ and concentrated in vacuo. Purification by column chromatography (petroleum ether/ethyl acetate $=40: 1)$ afforded $\mathbf{1 2 b}(602$ $\mathrm{mg}, 90 \%)$ as a colorless liquid.

12b: $[\alpha]^{21}{ }_{\mathrm{D}}-59.0\left(c 1.00, \mathrm{CHCl}_{3}\right) ;{ }^{1} \mathrm{H}$ NMR $\left(400 \mathrm{MHz}, \mathrm{CDCl}_{3}\right) \delta 6.91(\mathrm{~d}, J=8.4 \mathrm{~Hz}, 1 \mathrm{H}), 6.35$ $(\mathrm{d}, J=8.0 \mathrm{~Hz}, 1 \mathrm{H}), 6.06-6.15(\mathrm{~m}, 1 \mathrm{H}), 5.46(\mathrm{dd}, J=1.2 \mathrm{~Hz}, 17.2 \mathrm{~Hz}, 1 \mathrm{H}), 5.36(\mathrm{dt}, J=1.2 \mathrm{~Hz}$, $7.2 \mathrm{~Hz}, 1 \mathrm{H}), 5.29$ (d, $J=10.4 \mathrm{~Hz}, 1 \mathrm{H}), 4.53$ (d, $J=5.2 \mathrm{~Hz}, 2 \mathrm{H}), 3.21-3.32(\mathrm{~m}, 2 \mathrm{H}), 2.80-2.91(\mathrm{~m}$, $2 \mathrm{H}), 1.96-2.03(\mathrm{~m}, 1 \mathrm{H}), 1.85-1.92(\mathrm{~m}, 1 \mathrm{H}), 1.71-1.76(\mathrm{~m}, 9 \mathrm{H}), 1.59-1.66(\mathrm{~m}, 1 \mathrm{H}), 1.37(\mathrm{sept}, J=$ 
$6.8 \mathrm{~Hz}, 1 \mathrm{H}), 1.26(\mathrm{dd}, J=3.2 \mathrm{~Hz}, 8.0 \mathrm{~Hz}, 1 \mathrm{H}), 0.93-0.99(\mathrm{~m}, 6 \mathrm{H}), 0.89(\mathrm{t}, J=3.6 \mathrm{~Hz}, 1 \mathrm{H}), 0.42$ $(\mathrm{dd}, J=5.2 \mathrm{~Hz}, 7.6 \mathrm{~Hz}, 1 \mathrm{H}) ;{ }^{13} \mathrm{C} \mathrm{NMR}\left(100 \mathrm{MHz}, \mathrm{CDCl}_{3}\right) \delta 155.02,153.41,133.80,131.16$, $126.11,123.60,122.30,116.66,110.59,101.72,83.92$, 68.54, 33.29, 32.92, 32.61, 30.00, 29.20, 28.17, 25.83, 24.64, 19.61, 19.59, 17.73, 17.49, 11.67; HRMS (ESIMS) calculated for $\mathrm{C}_{25} \mathrm{H}_{35} \mathrm{O}_{2}$ $[\mathrm{M}+\mathrm{H}]^{+} 367.2632$, found 367.2635 .

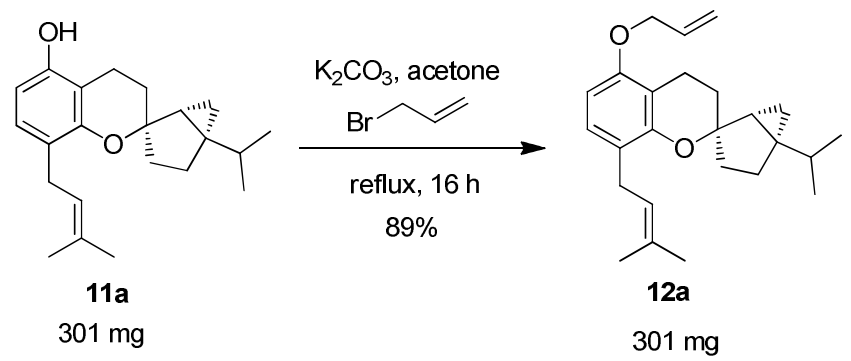

12a: $[\alpha]^{21}{ }_{\mathrm{D}}-10.0\left(c 1.00, \mathrm{CHCl}_{3}\right) ;{ }^{1} \mathrm{H}$ NMR $\left(400 \mathrm{MHz}, \mathrm{CDCl}_{3}\right) \delta 6.90(\mathrm{~d}, J=8.0 \mathrm{~Hz}, 1 \mathrm{H}), 6.34$ (d, $J=8.4 \mathrm{~Hz}, 1 \mathrm{H}), 6.05-6.14(\mathrm{~m}, 1 \mathrm{H}), 5.45(\mathrm{dd}, J=0.8 \mathrm{~Hz}, 17.2 \mathrm{~Hz}, 1 \mathrm{H}), 5.37(\mathrm{t}, J=7.2 \mathrm{~Hz}, 1 \mathrm{H})$, $5.28(\mathrm{~d}, J=10.4 \mathrm{~Hz}, 1 \mathrm{H}), 4.52(\mathrm{~d}, J=4.8 \mathrm{~Hz}, 2 \mathrm{H}), 3.20-3.33(\mathrm{~m}, 2 \mathrm{H}), 2.80-2.87(\mathrm{~m}, 1 \mathrm{H})$, 2.64-2.71 (m, 1H), 1.97-2.07 (m, 1H), 1.85-1.93 (m, 2H), 1.78-1.83 (m, 1H), $1.76(\mathrm{~s}, 3 \mathrm{H}), 1.72(\mathrm{~s}$, $3 \mathrm{H}), 1.63$ (dd, $J=8.0 \mathrm{~Hz}, 12.0 \mathrm{~Hz}, 1 \mathrm{H}), 1.42$ (sept, $J=6.8 \mathrm{~Hz}, 1 \mathrm{H}), 1.27-1.30$ (m, 1H), 1.23 (td, $J$ $=2.8 \mathrm{~Hz}, 11.2 \mathrm{~Hz}, 1 \mathrm{H}), 1.04(\mathrm{~d}, J=6.8 \mathrm{~Hz}, 3 \mathrm{H}), 0.97(\mathrm{~d}, J=6.8 \mathrm{~Hz}, 3 \mathrm{H}), 0.45(\mathrm{dd}, J=5.2 \mathrm{~Hz}, 8.0$ $\mathrm{Hz}, 1 \mathrm{H}), 0.34(\mathrm{t}, J=4.4 \mathrm{~Hz}, 1 \mathrm{H}) ;{ }^{13} \mathrm{C} \mathrm{NMR}\left(100 \mathrm{MHz}, \mathrm{CDCl}_{3}\right) \delta 155.0,152.2,133.9,131.5$, 126.0, 123.5, 122.4, 116.6, 110.9, 101.8, 86.3, 68.7, 34.2, 32.7, 32.6, 30.6, 28.2, 27.8, 25.8, 25.4, 20.2, 19.7, 18.6, 17.7, 12.5; HRMS (ESIMS) calculated for $\mathrm{C}_{25} \mathrm{H}_{35} \mathrm{O}_{2}[\mathrm{M}+\mathrm{H}]^{+} 367.2632$, found 367.2638 .

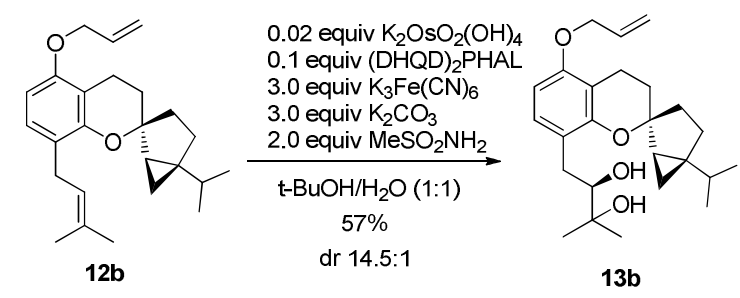

A mixture of $\mathrm{K}_{2} \mathrm{CO}_{3}(52 \mathrm{mg}, 0.38 \mathrm{mmol}), \mathrm{K}_{3} \mathrm{Fe}(\mathrm{CN})_{6}(123 \mathrm{mg}, 0.38 \mathrm{mmol}), \mathrm{K}_{2} \mathrm{OsO}_{2}(\mathrm{OH})_{4}(1$ $\mathrm{mg}, 0.0025 \mathrm{mmol})$ and (DHQD) 2 PHAL $(10 \mathrm{mg}, 0.013 \mathrm{mmol})$ in 1:1 tert-butyl alcohol-water was cooled to $0{ }^{\circ} \mathrm{C}$. The resulting suspension was treated with the $\mathbf{1 2 b}(46 \mathrm{mg}, 0.13 \mathrm{mmol})$ and $\mathrm{MeSO}_{2} \mathrm{NH}_{2}(24 \mathrm{mg}, 0.25 \mathrm{mmol})$, stirred at $0{ }^{\circ} \mathrm{C}$ for $16 \mathrm{~h}$. The reaction mixture quenched by addition of $\mathrm{Na}_{2} \mathrm{~S}_{2} \mathrm{O}_{3}$ and extracted with ethyl acetate. The combined organic extracts were washed with water, brine, successively, dried over $\mathrm{Na}_{2} \mathrm{SO}_{4}$ and concentrated in vacuo. Purification by column chromatography (petroleum ether/ethyl acetate $=2.5: 1)$ afforded $\mathbf{1 3 b}(29 \mathrm{mg}, 57 \%)$ as a colorless liquid.

13b: $[\alpha]^{21}{ }_{\mathrm{D}}-8.0\left(c\right.$ 1.00, MeOH); ${ }^{1} \mathrm{H}$ NMR $\left(400 \mathrm{MHz}, \mathrm{CDCl}_{3}\right) \delta 6.91(\mathrm{~d}, J=8.0 \mathrm{~Hz}, 1 \mathrm{H}), 6.36$ $(\mathrm{d}, J=8.0 \mathrm{~Hz}, 1 \mathrm{H}), 6.03-6.12(\mathrm{~m}, 1 \mathrm{H}), 5.43(\mathrm{dd}, J=1.6 \mathrm{~Hz}, 17.2 \mathrm{~Hz}, 1 \mathrm{H}), 5.28(\mathrm{dd}, J=1.2 \mathrm{~Hz}$, $10.4 \mathrm{~Hz}, 1 \mathrm{H}), 4.52(\mathrm{~d}, J=5.2 \mathrm{~Hz}, 2 \mathrm{H}), 3.53-3.57(\mathrm{~m}, 1 \mathrm{H}), 2.96(\mathrm{~d}, J=3.6 \mathrm{~Hz}, 1 \mathrm{H}), 2.72-2.88(\mathrm{~m}$, $3 \mathrm{H}), 2.59(\mathrm{dd}, J=10.4 \mathrm{~Hz}, 13.6 \mathrm{~Hz}, 1 \mathrm{H}), 2.51(\mathrm{~s}, 1 \mathrm{H}), 1.93-2.00(\mathrm{~m}, 1 \mathrm{H}), 1.81-1.88(\mathrm{~m}, 1 \mathrm{H})$, $1.72-1.74(\mathrm{~m}, 3 \mathrm{H}), 1.48-1.55(\mathrm{~m}, 1 \mathrm{H}), 1.33-1.40(\mathrm{~m}, 1 \mathrm{H}), 1.23-1.29(\mathrm{~m}, 7 \mathrm{H}), 0.94(\mathrm{~d}, J=6.8 \mathrm{~Hz}$, $3 \mathrm{H}), 0.89(\mathrm{~d}, J=6.8 \mathrm{~Hz}, 3 \mathrm{H}), 0.84(\mathrm{t}, J=4.0 \mathrm{~Hz}, 1 \mathrm{H}), 0.46(\mathrm{dd}, J=5.2 \mathrm{~Hz}, 8.0 \mathrm{~Hz}, 1 \mathrm{H}) ;{ }^{13} \mathrm{C} \mathrm{NMR}$ 
$\left(100 \mathrm{MHz}, \mathrm{CDCl}_{3}\right) \delta 155.69,153.37,133.56,128.15,119.39,116.92,110.86,102.41,84.89,79.23$, 72.70, 68.65, 33.31, 32.94, 32.52, 32.48, 29.79, 29.59, 25.99, 24.75, 23.54, 19.58, 17.24, 11.56; HRMS (ESIMS) calculated for $\mathrm{C}_{25} \mathrm{H}_{37} \mathrm{O}_{4}[\mathrm{M}+\mathrm{H}]^{+} 401.2686$, found 401.2692.

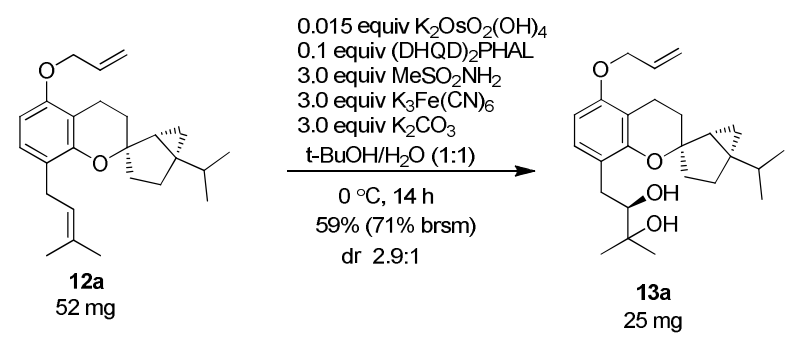

The equivalents of $\mathrm{K}_{2} \mathrm{OSO}_{2}(\mathrm{OH})_{4}$ and $\mathrm{MeSO}_{2} \mathrm{NH}_{2}$ are different from those for dihydroxylation of $\mathbf{1 2 b}$.

13a: $[\alpha]^{21}{ }_{\mathrm{D}}+19.0\left(c\right.$ 1.00, MeOH); ${ }^{1} \mathrm{H} \mathrm{NMR}\left(400 \mathrm{MHz}, \mathrm{CDCl}_{3}\right) \delta 6.93(\mathrm{~d}, J=8.4 \mathrm{~Hz}, 1 \mathrm{H}), 6.37$ $(\mathrm{d}, J=4.4 \mathrm{~Hz}, 1 \mathrm{H}), 6.02-6.12(\mathrm{~m}, 1 \mathrm{H}), 5.43(\mathrm{dd}, J=1.6 \mathrm{~Hz}, 17.2 \mathrm{~Hz}, 1 \mathrm{H}), 5.27$ (dd, $J=1.2 \mathrm{~Hz}$, $10.4 \mathrm{~Hz}, 1 \mathrm{H}), 4.52(\mathrm{~d}, J=5.2 \mathrm{~Hz}, 2 \mathrm{H}), 3.58(\mathrm{td}, J=2.8 \mathrm{~Hz}, 10.0 \mathrm{~Hz}, 1 \mathrm{H}), 2.95(\mathrm{~d}, J=3.2 \mathrm{~Hz}, 1 \mathrm{H})$, 2.65-2.84 (m, 4H), $2.47(\mathrm{~s}, 1 \mathrm{H}), 1.96-2.04(\mathrm{~m}, 1 \mathrm{H}), 1.86(\mathrm{t}, 6.8 \mathrm{~Hz}, 2 \mathrm{H}), 1.77$ (dd, J = 7.6 Hz, 18.0 $\mathrm{Hz}, 1 \mathrm{H}), 1.69(\mathrm{dd}, J=4.0 \mathrm{~Hz}, 8.0 \mathrm{~Hz}, 1 \mathrm{H}), 1.40-1.49(\mathrm{~m}, 1 \mathrm{H}), 1.26-1.33(\mathrm{~m}, 8 \mathrm{H}), 1.01(\mathrm{~d}, J=6.8$ $\mathrm{Hz}, 3 \mathrm{H}), 0.90(\mathrm{~d}, J=6.8 \mathrm{~Hz}, 3 \mathrm{H}), 0.45(\mathrm{dd}, J=5.2 \mathrm{~Hz}, 8.0 \mathrm{~Hz}, 1 \mathrm{H}), 0.29-0.31(\mathrm{~m}, 1 \mathrm{H}) ;{ }^{13} \mathrm{C}$ NMR $\left(100 \mathrm{MHz}, \mathrm{CDCl}_{3}\right) \delta 155.65,152.37,133.60,128.29,119.45,116.85,111.43,102.58,87.48,79.15$, 72.68, 68.69, 34.54, 33.36, 32.71, 32.30, 29.96, 28.17, 26.02, 25.98, 23.75, 20.09, 19.92, 18.42, 12.12; HRMS (ESIMS) calculated for $\mathrm{C}_{25} \mathrm{H}_{36} \mathrm{O}_{4} \mathrm{Na}[\mathrm{M}+\mathrm{Na}]^{+} 423.2506$, found 423.2502.

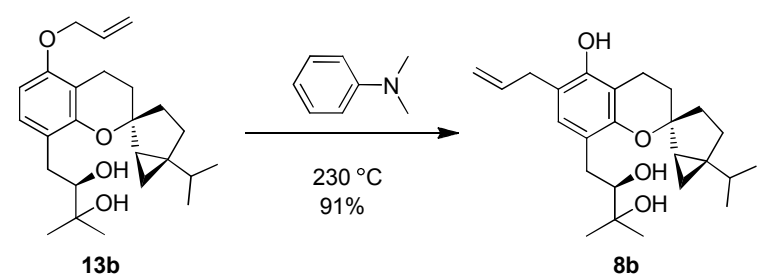

A solution of $\mathbf{1 3 b}(40 \mathrm{mg}, 0.10 \mathrm{mmol})$ in N,N-Dimethylaniline $(2.5 \mathrm{~mL})$ was heated at $230{ }^{\circ} \mathrm{C}$ in a sealed tube for $4 \mathrm{~h}$, then cooled to room temperature. The reaction was added a solution of 2 $\mathrm{N} \mathrm{HCl}(5 \mathrm{~mL})$ and extracted with ethyl acetate. The combined organic extracts were washed with $2 \mathrm{~N} \mathrm{HCl}$ (8 times), water, brine, successively, dried over $\mathrm{Na}_{2} \mathrm{SO}_{4}$ and concentrated in vacuo. Purification by column chromatography (petroleum ether/ethyl acetate $=3: 1)$ afforded $\mathbf{8 b}(37 \mathrm{mg}$, $91 \%)$ as a colorless liquid.

8b: $[\alpha]^{21}{ }_{\mathrm{D}}-6.0\left(c\right.$ 1.00, MeOH); ${ }^{1} \mathrm{H}$ NMR $\left(400 \mathrm{MHz}, \mathrm{CDCl}_{3}\right) \delta 6.73(\mathrm{~s}, 1 \mathrm{H}), 6.00-6.05(\mathrm{~m}, 1 \mathrm{H})$, 5.17-5.25 (m, 2H), $5.03(\mathrm{~s}, 1 \mathrm{H}), 3.54(\mathrm{~d}, J=10.4 \mathrm{~Hz}, 1 \mathrm{H}), 3.33(\mathrm{~d}, J=6.4 \mathrm{~Hz}, 2 \mathrm{H}), 2.97(\mathrm{~d}, J=$ $3.2 \mathrm{~Hz}, 1 \mathrm{H}), 2.67-2.84(\mathrm{~m}, 3 \mathrm{H}), 2.59$ (dd, $J=10.0,13.6 \mathrm{~Hz}, 1 \mathrm{H}), 2.49$ (s, 1H), 1.94-2.01 (m, 1H), $1.85-1.90(\mathrm{~m}, 1 \mathrm{H}), 1.68-1.74(\mathrm{~m}, 3 \mathrm{H}), 1.48-1.49(\mathrm{~m}, 1 \mathrm{H}), 1.35-1.39(\mathrm{~m}, 1 \mathrm{H}), 1.22-1.29(\mathrm{~m}, 7 \mathrm{H})$, $0.94(\mathrm{~d}, J=6.8 \mathrm{~Hz}, 3 \mathrm{H}), 0.89(\mathrm{~d}, J=6.8 \mathrm{~Hz}, 3 \mathrm{H}), 0.84-0.86(\mathrm{~m}, 1 \mathrm{H}), 0.46(\mathrm{dd}, J=5.2,7.6 \mathrm{~Hz}$, $1 \mathrm{H}) ;{ }^{13} \mathrm{C} \mathrm{NMR}\left(100 \mathrm{MHz}, \mathrm{CDCl}_{3}\right) \delta 152.3,151.4,137.0,129.7,118.7,116.6,115.1,109.5,84.8$, 79.3, 72.7, 35.4, 33.3, 32.8, 32.5, 29.8, 29.6, 26.0, 24.8, 23.6, 19.6, 17.2, 11.6; HRMS (ESIMS) calculated for $\mathrm{C}_{25} \mathrm{H}_{37} \mathrm{O}_{4}[\mathrm{M}+\mathrm{H}]^{+} 401.2686$, found 401.2697 . 


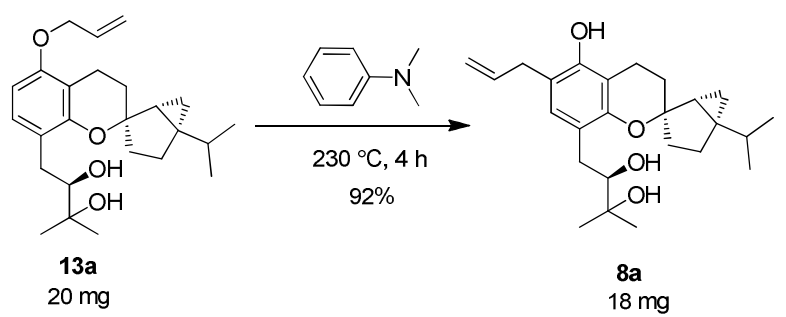

8a: $[\alpha]^{20}{ }_{D}-12.0\left(c\right.$ 1.00, MeOH); ${ }^{1} \mathrm{H}$ NMR $\left(400 \mathrm{MHz}, \mathrm{CDCl}_{3}\right) \delta 6.74(\mathrm{~s}, 1 \mathrm{H})$, 5.96-6.06 (m, $1 \mathrm{H}), 5.17-5.25(\mathrm{~m}, 2 \mathrm{H}), 5.01(\mathrm{~s}, 1 \mathrm{H}), 3.56-3.60(\mathrm{~m}, 1 \mathrm{H}), 3.34(\mathrm{~d}, J=6.4 \mathrm{~Hz}, 2 \mathrm{H}), 2.97(\mathrm{~d}, J=3.6$ $\mathrm{Hz}, 1 \mathrm{H}), 2.63-2.79(\mathrm{~m}, 4 \mathrm{H}), 2.44(\mathrm{~s}, 1 \mathrm{H}), 1.95-2.03(\mathrm{~m}, 1 \mathrm{H}), 1.88(\mathrm{t}, J=6.4 \mathrm{~Hz}, 2 \mathrm{H}), 1.77(\mathrm{dd}, J=$ $4.4 \mathrm{~Hz}, 14.4 \mathrm{~Hz}, 1 \mathrm{H}), 1.68(\mathrm{dd}, J=8.0 \mathrm{~Hz}, 12.0 \mathrm{~Hz}, 1 \mathrm{H}), 1.40-1.47(\mathrm{~m}, 1 \mathrm{H}), 1.27-1.33(\mathrm{~m}, 5 \mathrm{H})$, $1.25(\mathrm{~s}, 3 \mathrm{H}), 1.00(\mathrm{~d}, J=6.8 \mathrm{~Hz}, 3 \mathrm{H}), 0.90(\mathrm{~d}, J=6.8 \mathrm{~Hz}, 3 \mathrm{H}), 0.46(\mathrm{dd}, J=5.2 \mathrm{~Hz}, 8.0 \mathrm{~Hz}, 1 \mathrm{H})$, 0.29-0.31 (m, 1H) ${ }^{13} \mathrm{C}_{\mathrm{NMR}}\left(100 \mathrm{MHz}, \mathrm{CDCl}_{3}\right) \delta 151.3,151.3,137.0 .129 .8,118.7,116.6,115.2$, 109.9, 87.3, 79.3, 72.6, 35.4, 34.6, 33.4, 32.6, 32.4, 29.9, 28.2, 26.0, 25.9, 23.8, 20.1, 20.0, 18.4, 12.3; HRMS (ESIMS) calculated for $\mathrm{C}_{25} \mathrm{H}_{36} \mathrm{O}_{4} \mathrm{Na}[\mathrm{M}+\mathrm{Na}]^{+} 423.2506$, found 423.2505 .
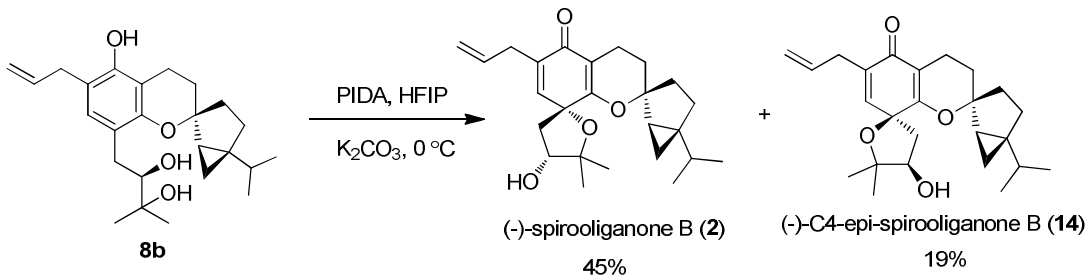

PIDA (20 mg, $0.063 \mathrm{mmol})$ was added to a stirred solution of $\mathbf{8 b}(25 \mathrm{mg}, 0.063 \mathrm{mmol})$ and $\mathrm{K}_{2} \mathrm{CO}_{3}(26 \mathrm{mg}, 0.19 \mathrm{mmol})$ in HFIP $(1.2 \mathrm{~mL})$ at $0{ }^{\circ} \mathrm{C}$ under $\mathrm{Ar}$ and the reaction mixture was stirred at the same temperature. After 10 minutes, the mixture was quenched by the addition of sat. aq. $\mathrm{NaHCO}_{3}$ and extracted with ethyl acetate. The combined organic extracts were washed with brine, dried over $\mathrm{Na}_{2} \mathrm{SO}_{4}$ and concentrated in vacuo. Purification by column chromatography (petroleum ether/ethyl acetate $=8: 1 \rightarrow 6: 1)$ afforded target molecule $(-)$-spirooliganone B (2) (11 $\mathrm{mg}, 45 \%)$ as a colorless liquid and (-)-C4-epi-spirooliganone B (14) (5 mg, 19\%).

(-)-spirooliganone B (2): $[\alpha]^{20}{ }_{\mathrm{D}}-54.0(c 1.00, \mathrm{MeOH}) ;{ }^{1} \mathrm{H}$ NMR $\left(400 \mathrm{MHz}\right.$, Acetone- $\left.\mathrm{d}_{6}\right) \delta 6.62$ (t, $J=1.2 \mathrm{~Hz}, 1 \mathrm{H}), 5.85$ (ddt, $J=17.0 \mathrm{~Hz}, 10.0 \mathrm{~Hz}, 7.0 \mathrm{~Hz}, 1 \mathrm{H}), 5.05$ (d, $J=17.2 \mathrm{~Hz}, 1 \mathrm{H}), 4.99$ (d, $J=10.4 \mathrm{~Hz}, 1 \mathrm{H}), 4.35-4.39(\mathrm{~m}, 1 \mathrm{H}), 4.31-4.32(\mathrm{~m}, 1 \mathrm{H}), 2.96(\mathrm{dd}, J=1.2 \mathrm{~Hz}, 6.8 \mathrm{~Hz}, 2 \mathrm{H}), 2.50$ $(\mathrm{dd}, J=6.0 \mathrm{~Hz}, 13.2 \mathrm{~Hz}, 1 \mathrm{H}), 2.26-2.41(\mathrm{~m}, 2 \mathrm{H}), 2.01(\mathrm{dd}, J=5.2 \mathrm{~Hz}, 13.2 \mathrm{~Hz}, 1 \mathrm{H}), \quad 1.83-1.90$ (m, 1H), 1.70-1.79 (m, 4H), 1.50-1.59 (m, 1H), 1.36-1.43 (m, 1H), $1.33(\mathrm{~s}, 3 \mathrm{H}), 1.26(\mathrm{~s}, 3 \mathrm{H}), 1.23$ $(\mathrm{dd}, J=3.6 \mathrm{~Hz}, 8.0 \mathrm{~Hz}, 1 \mathrm{H}), 0.95(\mathrm{~d}, J=6.8 \mathrm{~Hz}, 3 \mathrm{H}), 0.91(\mathrm{~d}, J=6.8 \mathrm{~Hz}, 3 \mathrm{H}), 0.88(\mathrm{dd}, J=4.0$ $\mathrm{Hz}, 4.8 \mathrm{~Hz}, 1 \mathrm{H}), 0.48(\mathrm{dd}, J=5.2 \mathrm{~Hz}, 8.0 \mathrm{~Hz}, 1 \mathrm{H}) ;{ }^{13} \mathrm{C}$ NMR $\left(100 \mathrm{MHz}\right.$, Acetone-d $\left.\mathrm{d}_{6}\right) \delta 185.56$, 168.99, 144.80, 137.14, 133.61, 116.43, 109.48, 87.45, 85.95, 78.86, 77.87, 44.62, 34.18, 33.93, $33.27,33.11,30.68,30.39,28.00,25.32,24.16,19.92,19.86,16.55,12.51$; HRMS (ESIMS) calculated for $\mathrm{C}_{25} \mathrm{H}_{35} \mathrm{O}_{4}[\mathrm{M}+\mathrm{H}]^{+} 399.2530$, found 399.2535 .

(-)-C4-epi-spirooliganone B (14): $[\alpha]^{20}{ }_{\mathrm{D}}-63.0(c$ 1.00, $\mathrm{MeOH}) ;{ }^{1} \mathrm{H} \mathrm{NMR}\left(400 \mathrm{MHz}, \mathrm{CDCl}_{3}\right) \delta$ $6.21(\mathrm{~s}, 1 \mathrm{H}), 5.78-5.88(\mathrm{~m}, 1 \mathrm{H}), 5.07-5.12(\mathrm{~m}, 2 \mathrm{H}), 4.13-4.16(\mathrm{~m}, 1 \mathrm{H}), 3.99-4.03(\mathrm{~m}, 1 \mathrm{H})$, 2.99-3.09 (m, 2H), $2.64(\mathrm{dd}, J=6.0 \mathrm{~Hz}, 14.8 \mathrm{~Hz}, 1 \mathrm{H}), 2.49-2.56(\mathrm{~m}, 1 \mathrm{H}), 2.21-2.32(\mathrm{~m}, 2 \mathrm{H})$, 1.69-1.89 (m, 5H), 1.36-1.43 (m, 5H), $1.31(\mathrm{~s}, 3 \mathrm{H}), 1.21(\mathrm{dd}, J=3.6 \mathrm{~Hz}, 8.0 \mathrm{~Hz}, 1 \mathrm{H}), 0.89-0.95(\mathrm{~m}$, $7 \mathrm{H}), 0.59(\mathrm{dd}, J=5.2 \mathrm{~Hz}, 8.0 \mathrm{~Hz}, 1 \mathrm{H}) ;{ }^{13} \mathrm{C} \mathrm{NMR}\left(150 \mathrm{MHz}, \mathrm{CDCl}_{3}\right) \delta 185.39,165.73,142.64$, 
135.20, 134.62, 116.95, 109.79, 88.45, 87.84, 78.95, 77.71, 44.07, 33.34, 33.00, 32.30, 31.07, $30.08,29.15,28.29,24.98,23.04,19.51,19.49,15.72,11.60$; HRMS (ESIMS) calculated for $\mathrm{C}_{25} \mathrm{H}_{35} \mathrm{O}_{4}[\mathrm{M}+\mathrm{H}]^{+} 399.2530$, found 399.2535.
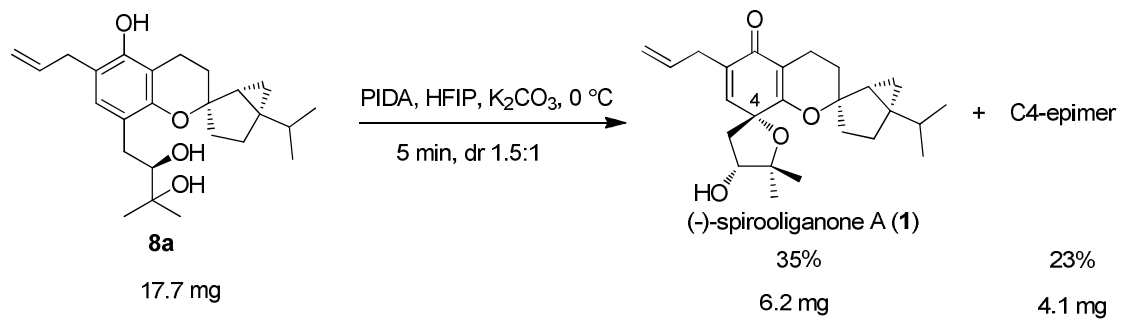

(-)-spirooliganone A (1): $[\alpha]^{20}{ }_{\mathrm{D}}-13.0(c 1.00, \mathrm{MeOH}) ;{ }^{1} \mathrm{H}$ NMR $\left(400 \mathrm{MHz}\right.$, Acetone- $\left.\mathrm{d}_{6}\right) \delta ; 6.64$ (s, 1H), $5.85(\mathrm{ddt}, J=17.2 \mathrm{~Hz}, 10.4 \mathrm{~Hz}, 6.8 \mathrm{~Hz}, 1 \mathrm{H}), 5.05(\mathrm{~d}, J=17.2 \mathrm{~Hz}, 1 \mathrm{H}), 4.99$ (d, $J=10.4$ $\mathrm{Hz}, 1 \mathrm{H}), 4.36-4.40(\mathrm{~m}, 2 \mathrm{H}), 2.97$ (dd, $J=1.2 \mathrm{~Hz}, 6.8 \mathrm{~Hz}, 2 \mathrm{H}), 2.62(\mathrm{dd}, J=6.0 \mathrm{~Hz}, 12.8 \mathrm{~Hz}, 1 \mathrm{H})$, 2.27-2.30 (m, 2H), 1.95-2.02 (m, 1H), 2.03 (m, 1H, overlapped), 1.71-1.83 (m, 4H), 1.61 (sept, $J$ $=6.8 \mathrm{~Hz}, 1 \mathrm{H}), 1.34-1.43(\mathrm{~m}, 2 \mathrm{H}), 1.32(\mathrm{~s}, 3 \mathrm{H}) .1 .26(\mathrm{~s}, 3 \mathrm{H}), 1.02(\mathrm{~d}, J=6.8 \mathrm{~Hz}, 3 \mathrm{H}), 0.87(\mathrm{~d}, J=$ $6.8 \mathrm{~Hz}, 3 \mathrm{H}), 0.51(\mathrm{dd}, J=5.2 \mathrm{~Hz}, 8.0 \mathrm{~Hz}, 1 \mathrm{H}), 0.35(\mathrm{dd}, J=3.6 \mathrm{~Hz}, 5.2 \mathrm{~Hz}, 1 \mathrm{H}) ;{ }^{13} \mathrm{C}$ NMR $(100$ $\left.\mathrm{MHz}, \mathrm{CDCl}_{3}\right) \delta 185.5,167.5,144.8,137.2,133.6,116.4,110.2,90.1,85.8,79.0,77.9,44.5,35.5$, 34.3, 33.9, 32.9, 30.5, 28.7, 28.2, 27.9, 24.1, 20.8, 20.1, 17.7, 11.6; HRMS (ESIMS) calculated for $\mathrm{C}_{25} \mathrm{H}_{35} \mathrm{O}_{4}[\mathrm{M}+\mathrm{H}]^{+} 399.2530$, found 399.2528 . 
${ }^{1} \mathrm{H}$ and ${ }^{13} \mathrm{C}$ NMR spectra of new compounds

$\stackrel{2}{i}$
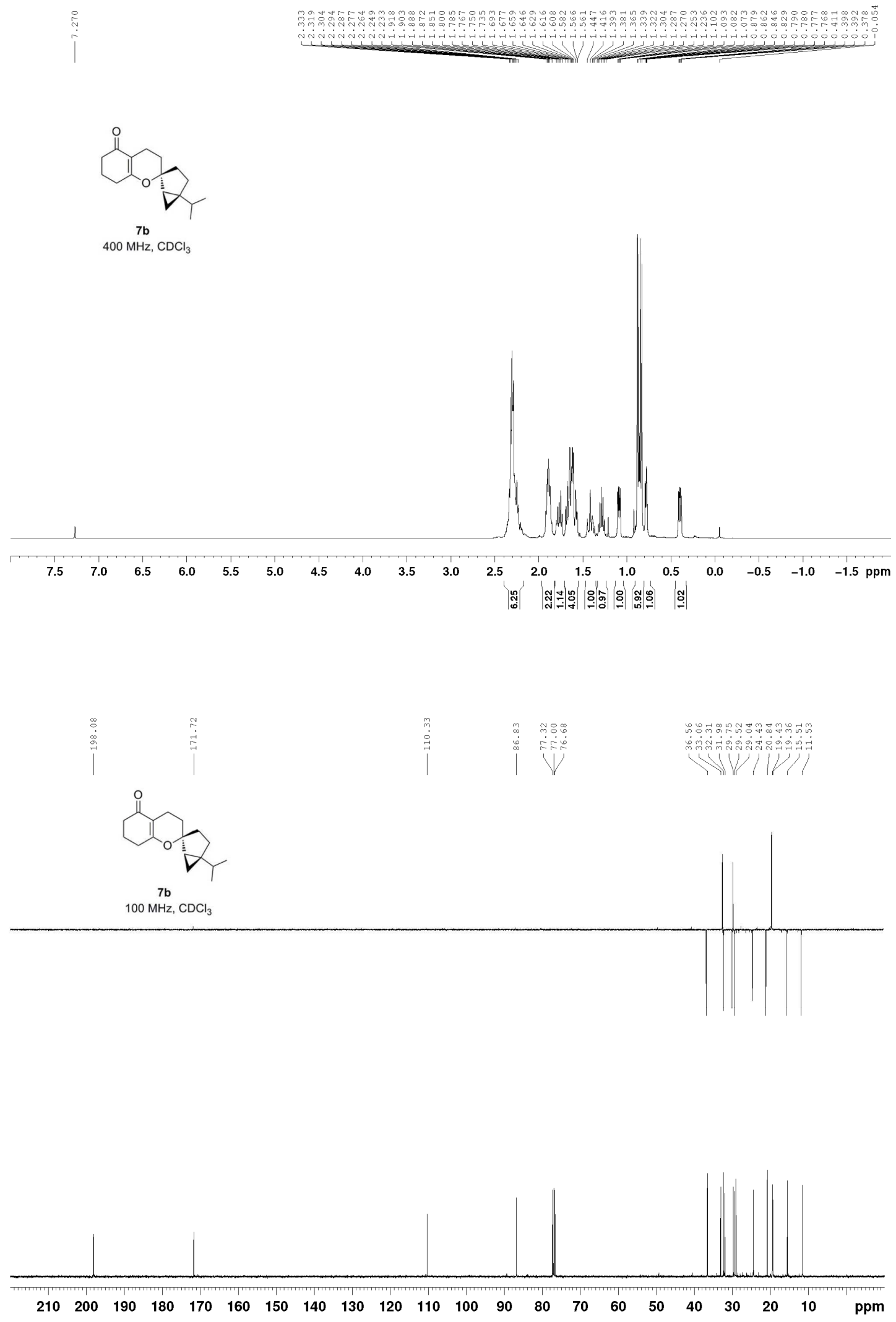

Sq 


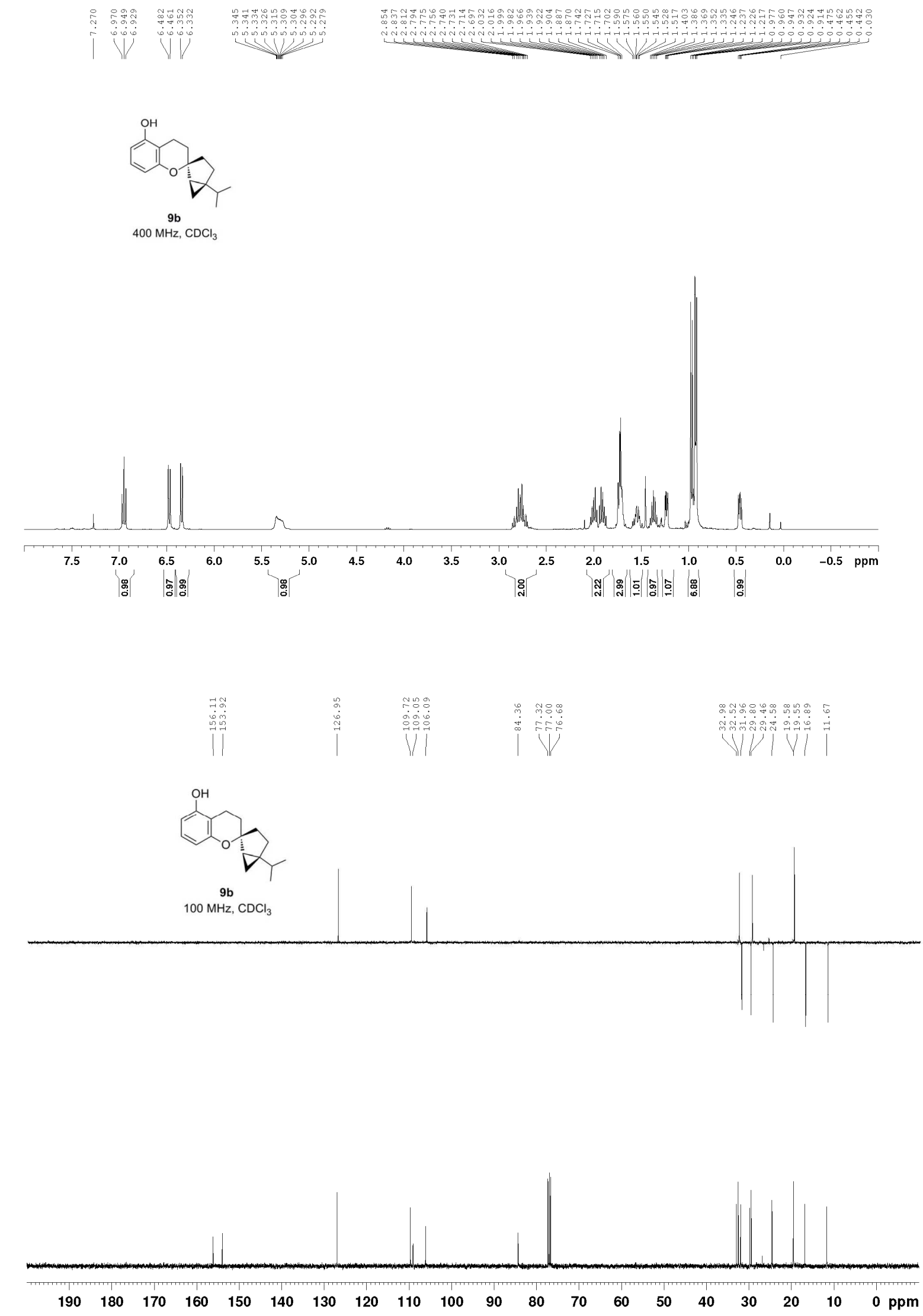




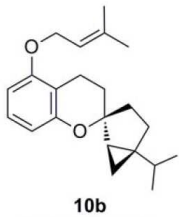

$400 \mathrm{MHz}, \mathrm{CDCl}_{3}$
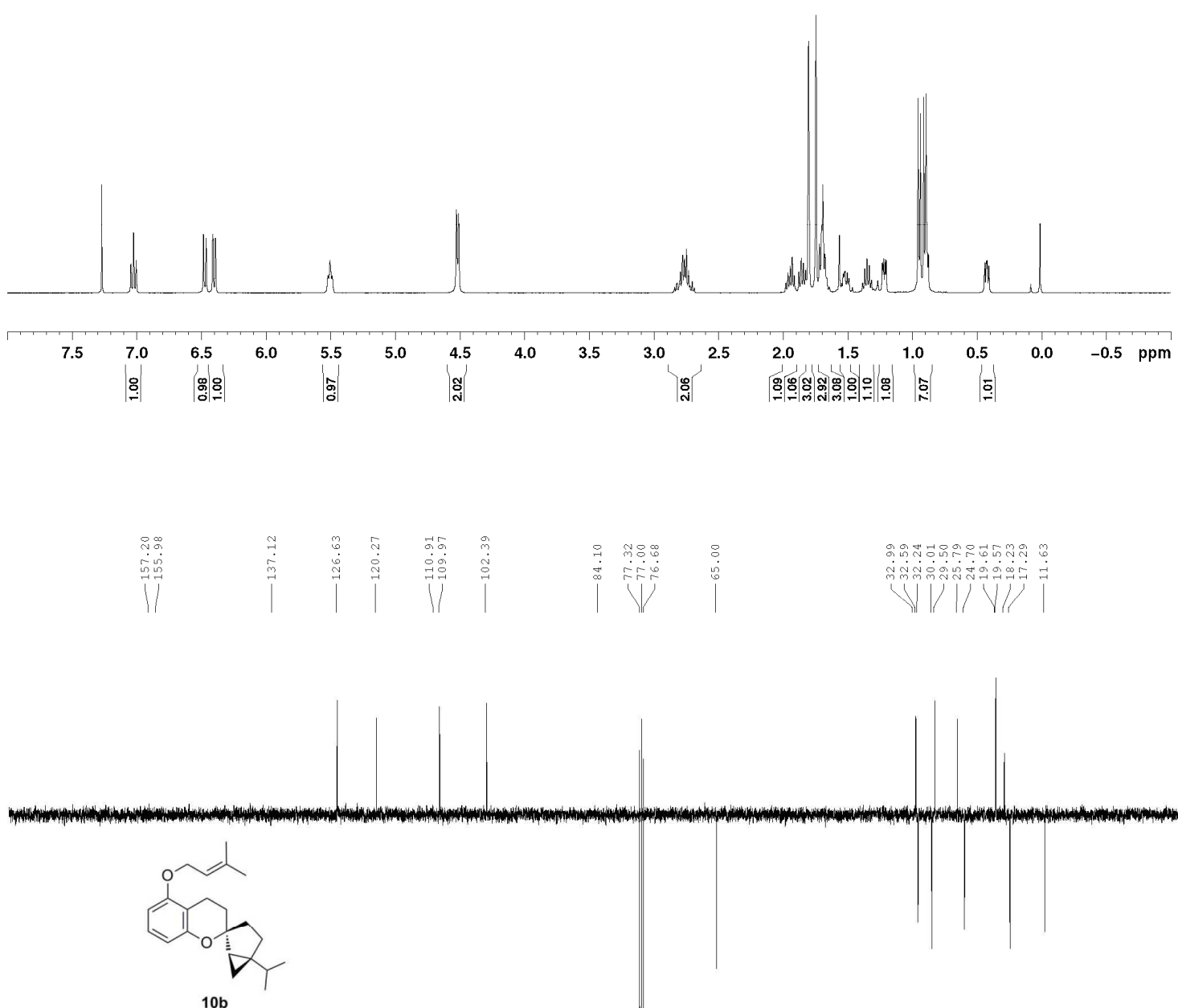

$100 \mathrm{MHz}, \mathrm{CDCl}_{3}$

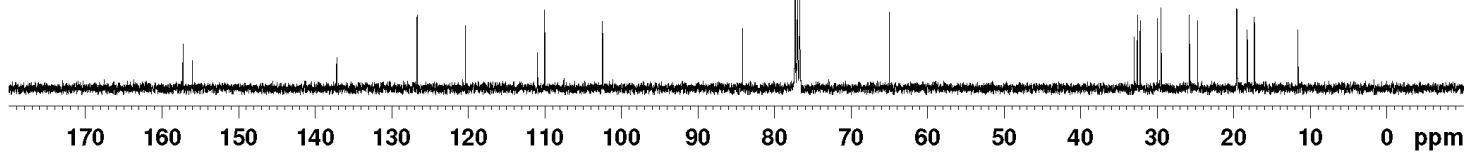



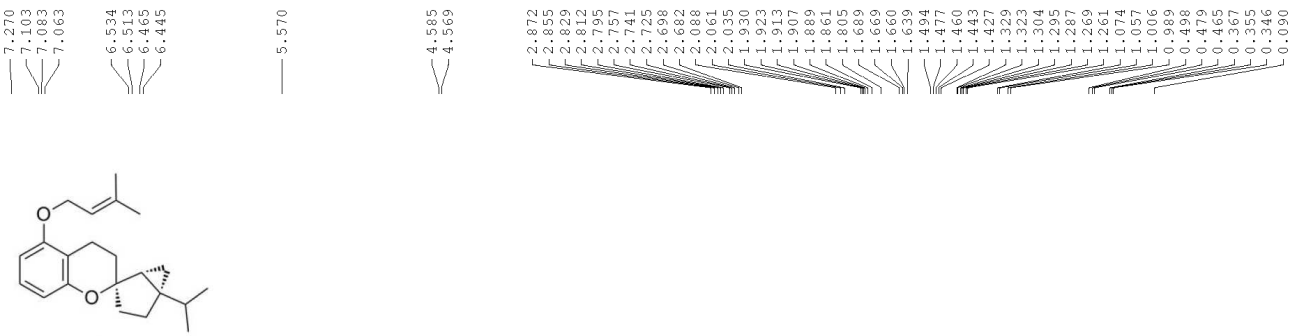

10a

$400 \mathrm{MHz}, \mathrm{CDCl}_{3}$
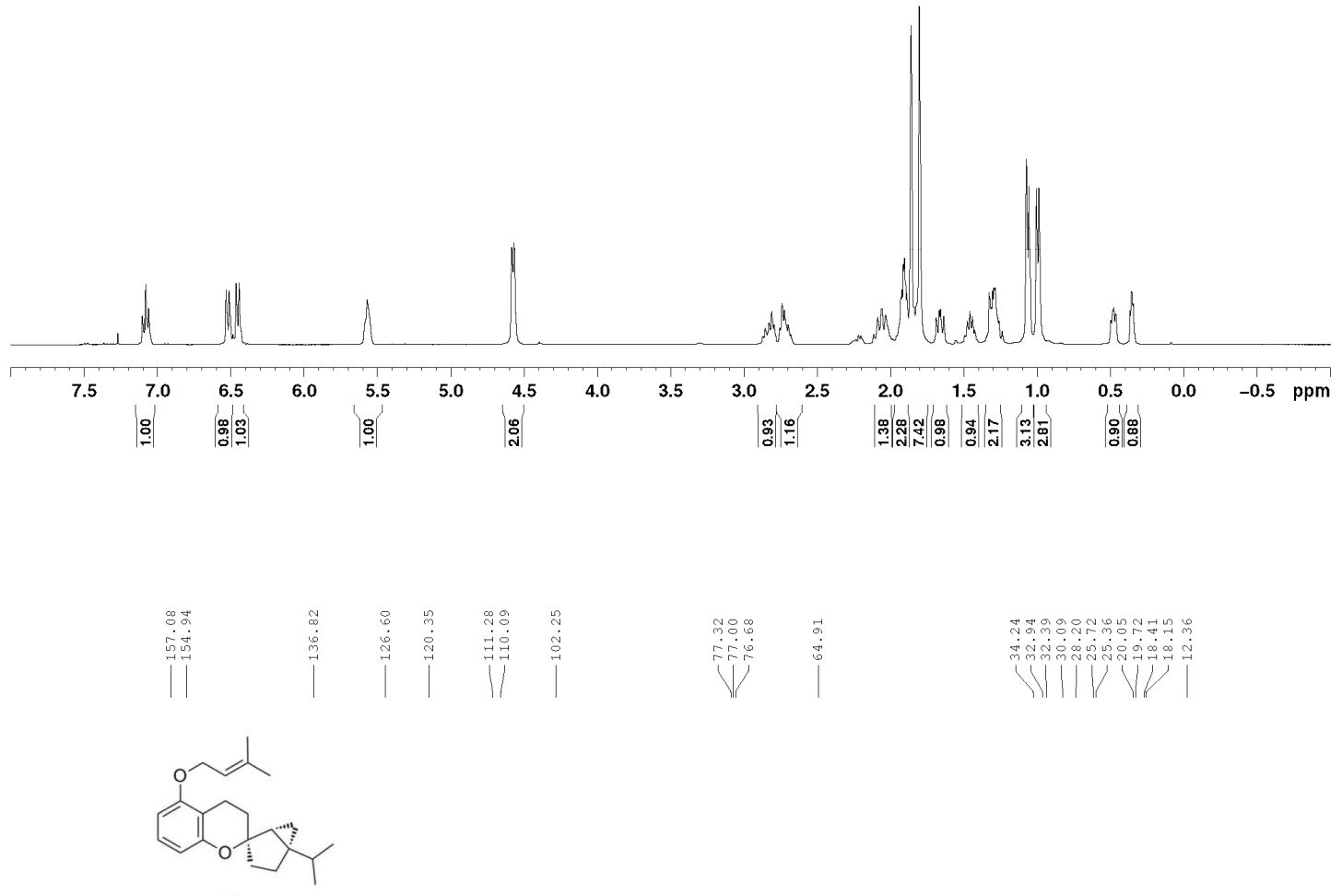

10a

$100 \mathrm{MHz}, \mathrm{CDCl}_{3}$
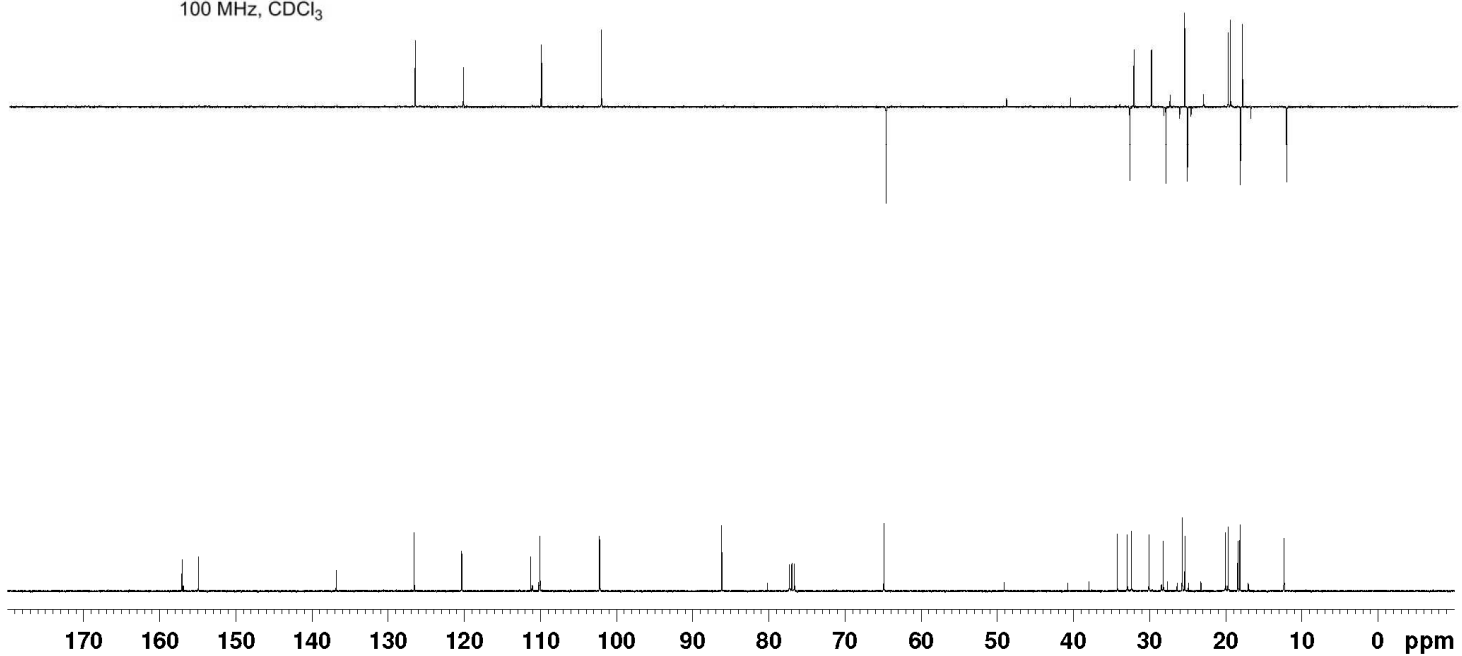


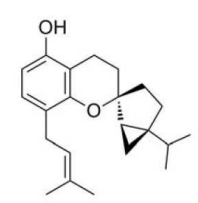

11b $400 \mathrm{MHz}, \mathrm{CDCl}_{3}$
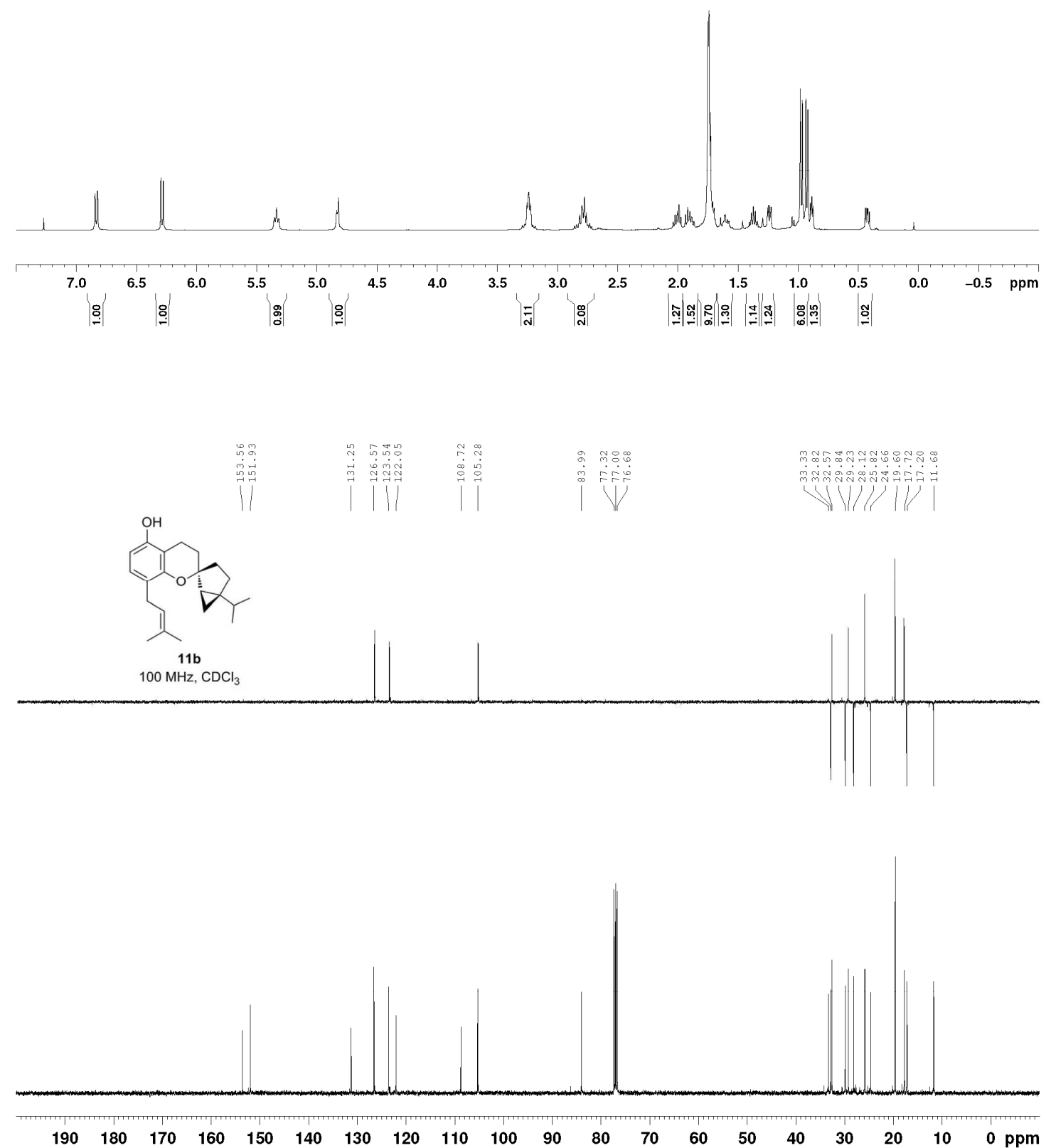

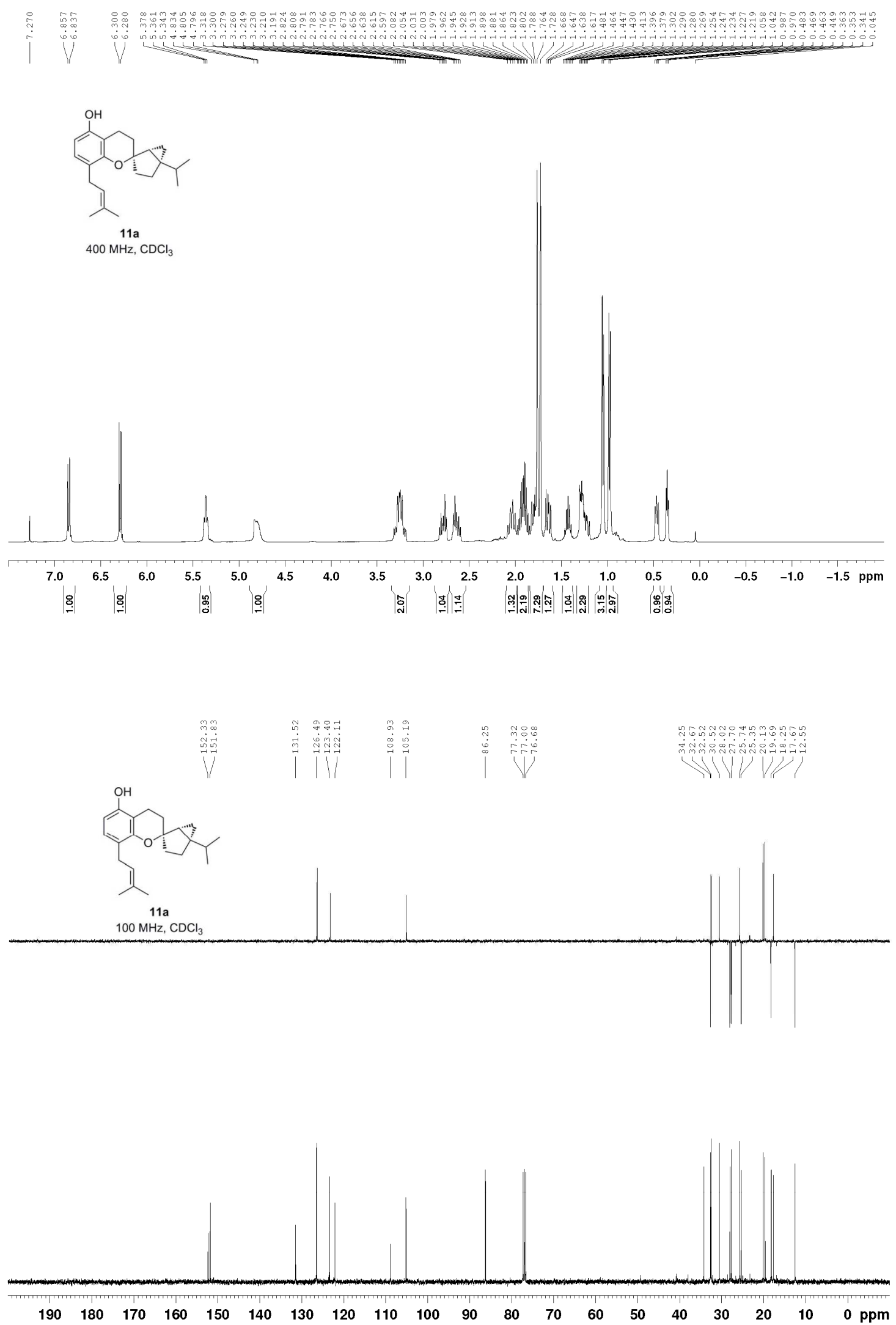


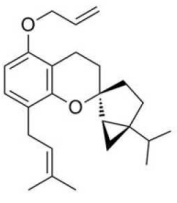

$12 \mathrm{~b}$

$400 \mathrm{MHz}, \mathrm{CDCl}_{3}$
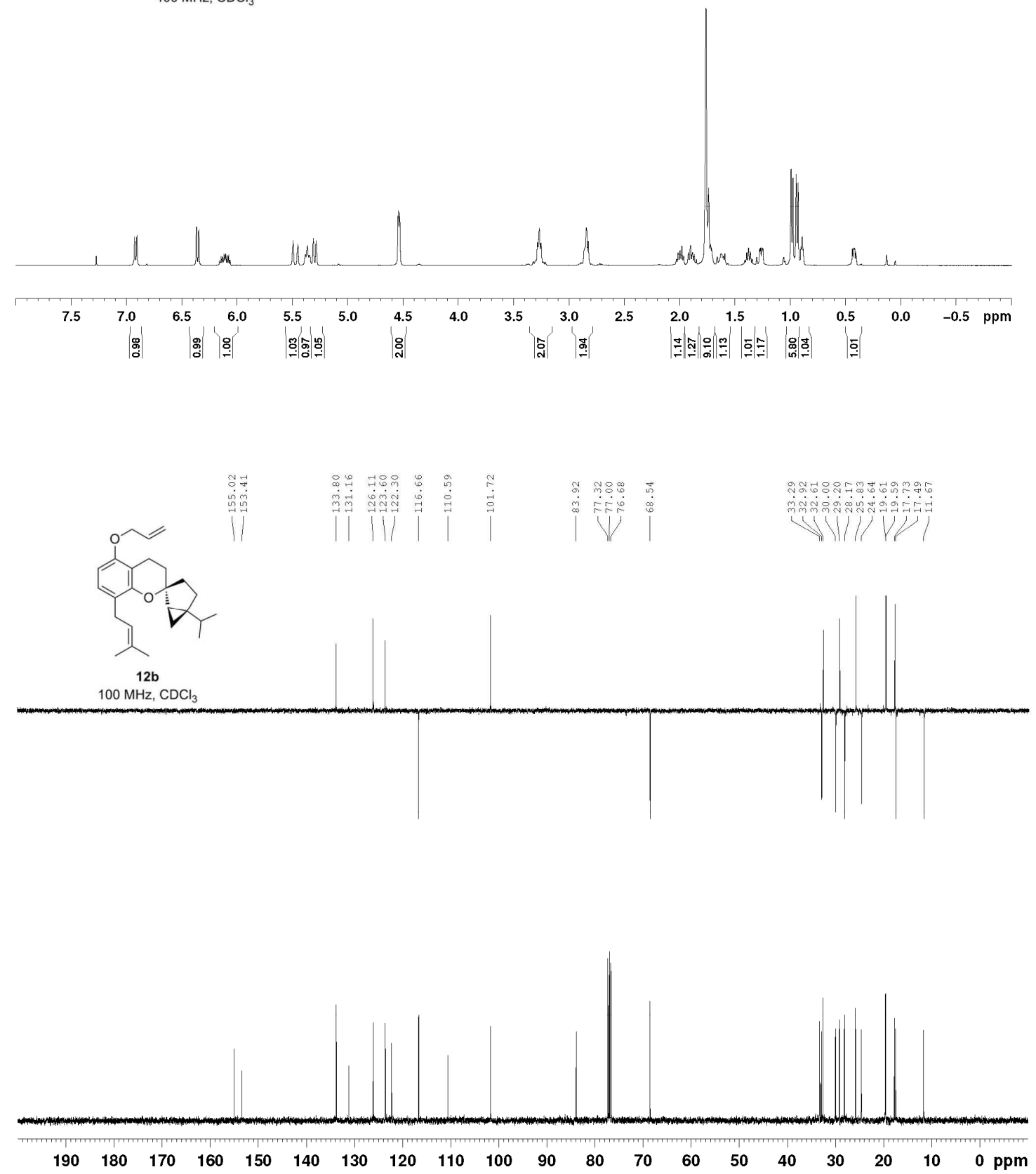


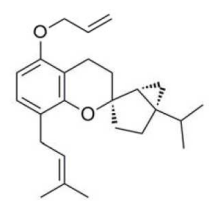

12a

$400 \mathrm{MHz}, \mathrm{CDCl}_{3}$
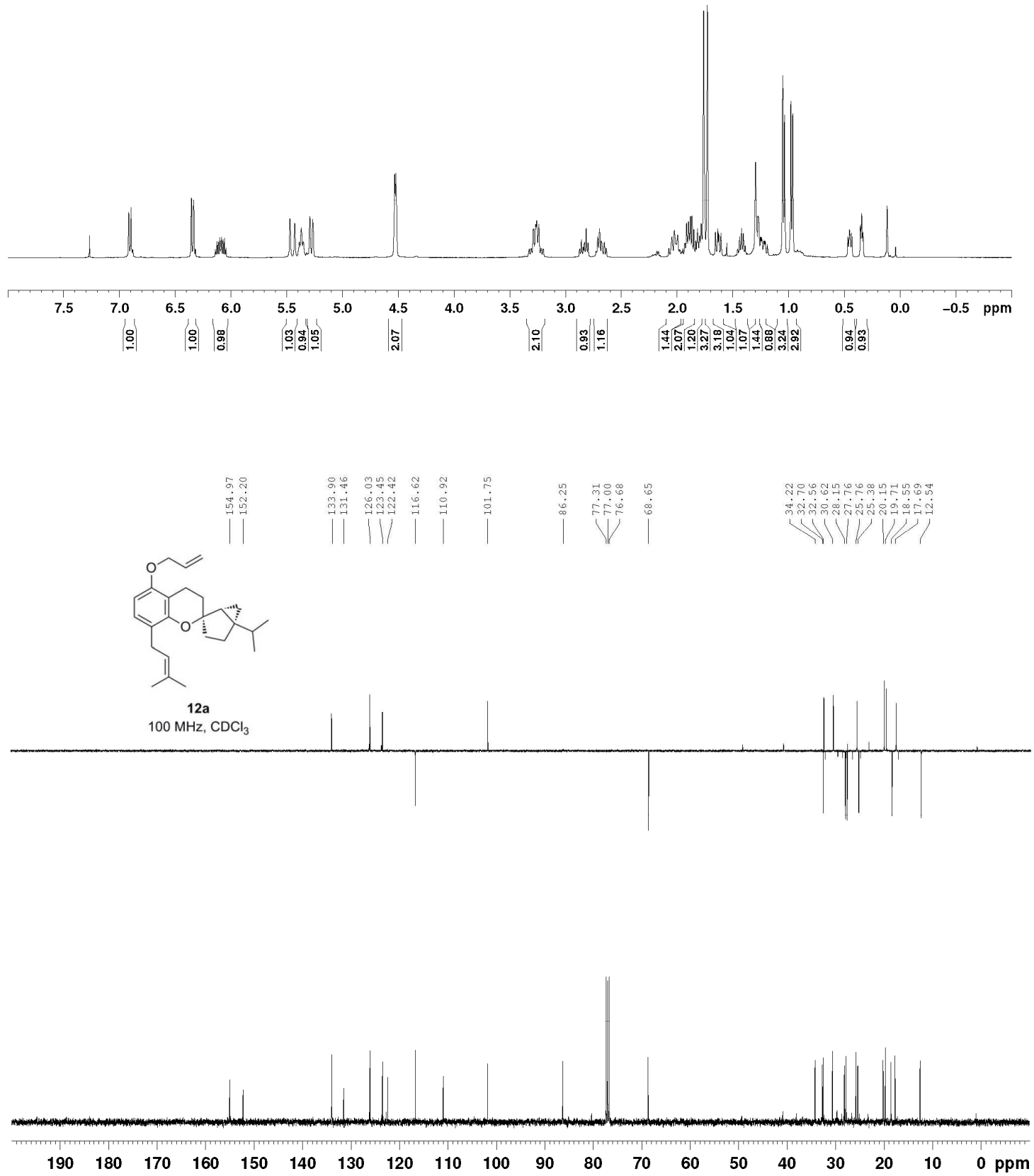


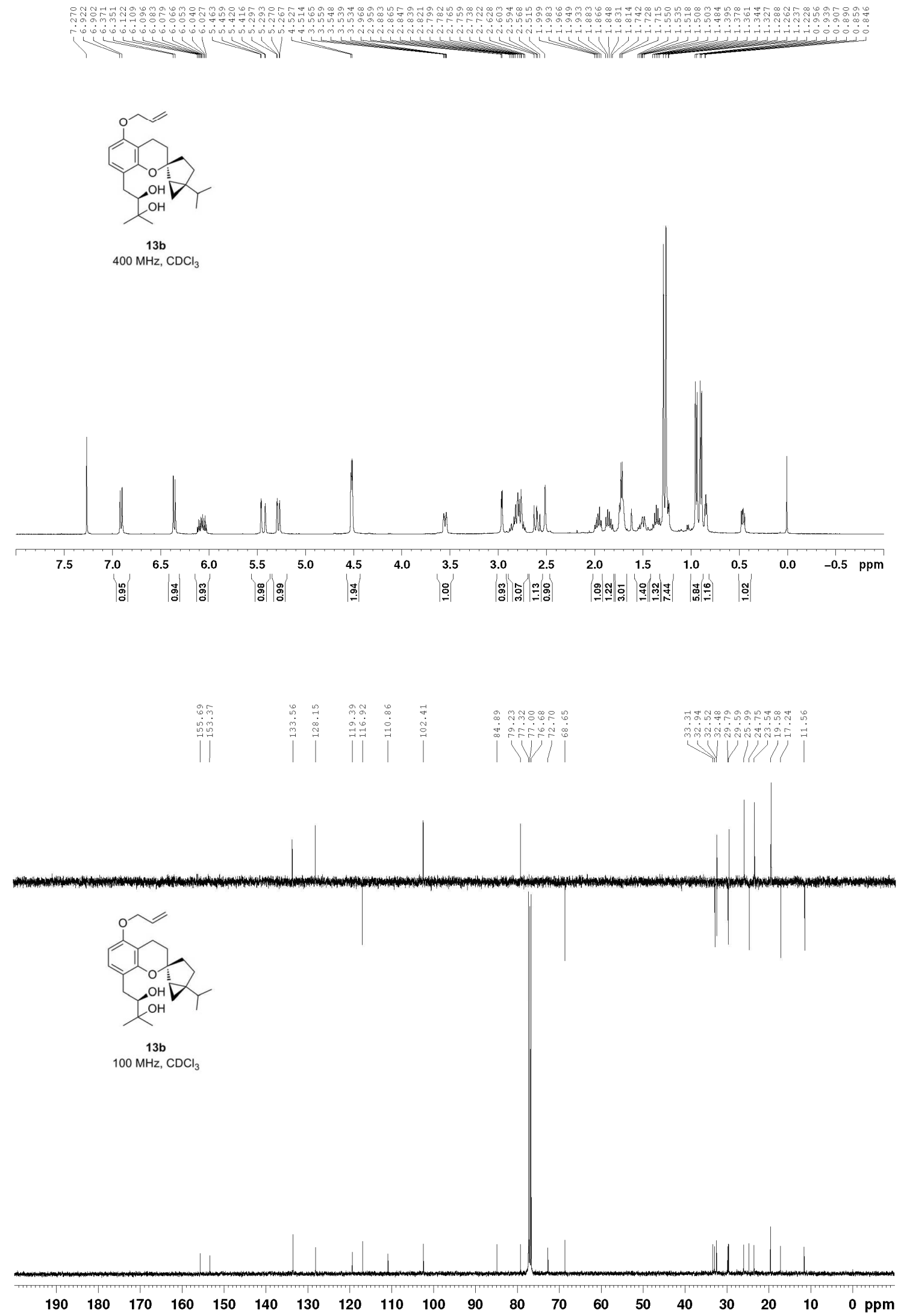




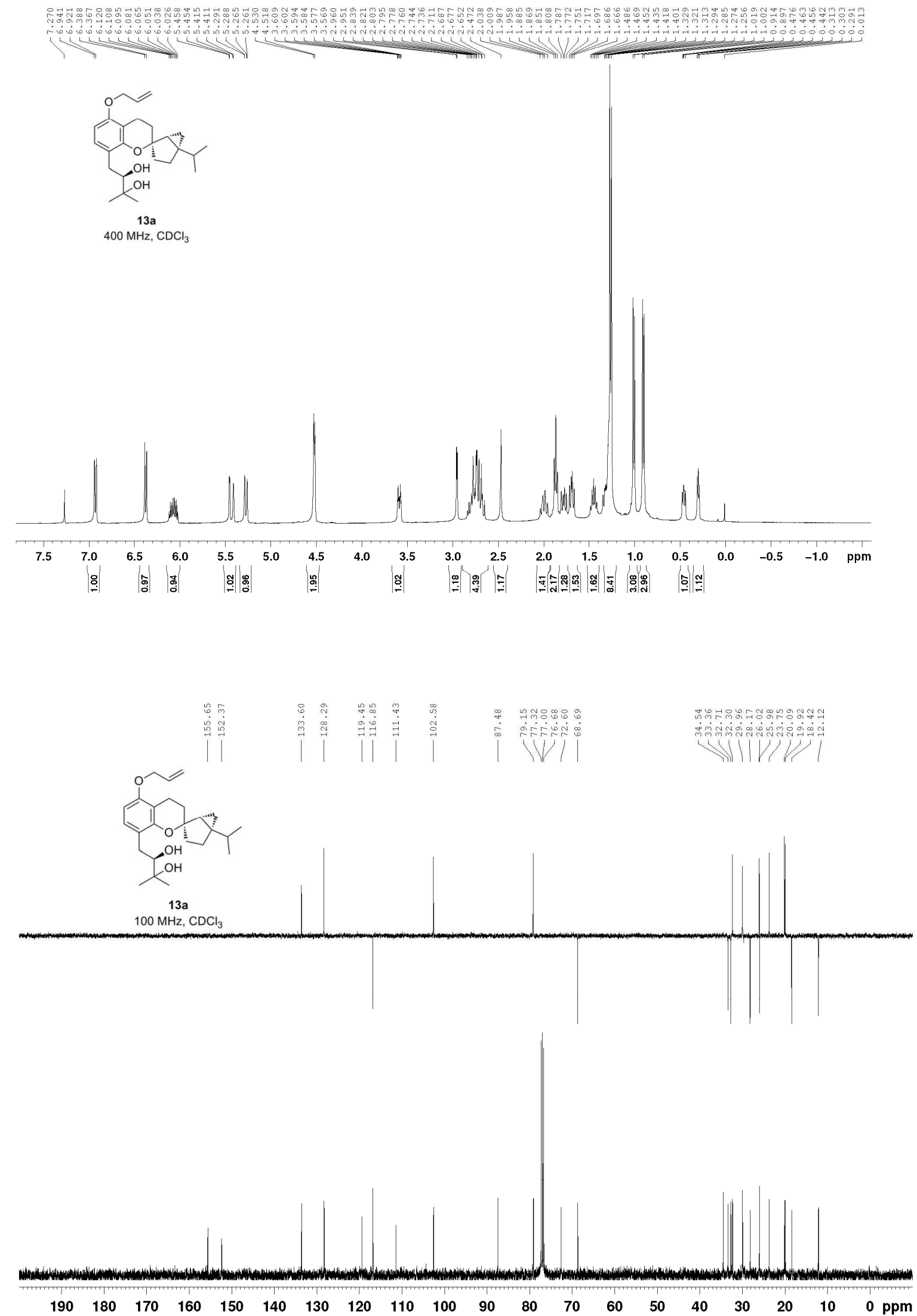




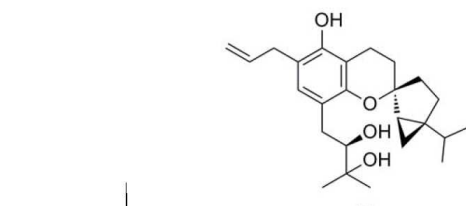

$400 \mathrm{MHz}, \mathrm{CDCl}_{3}$
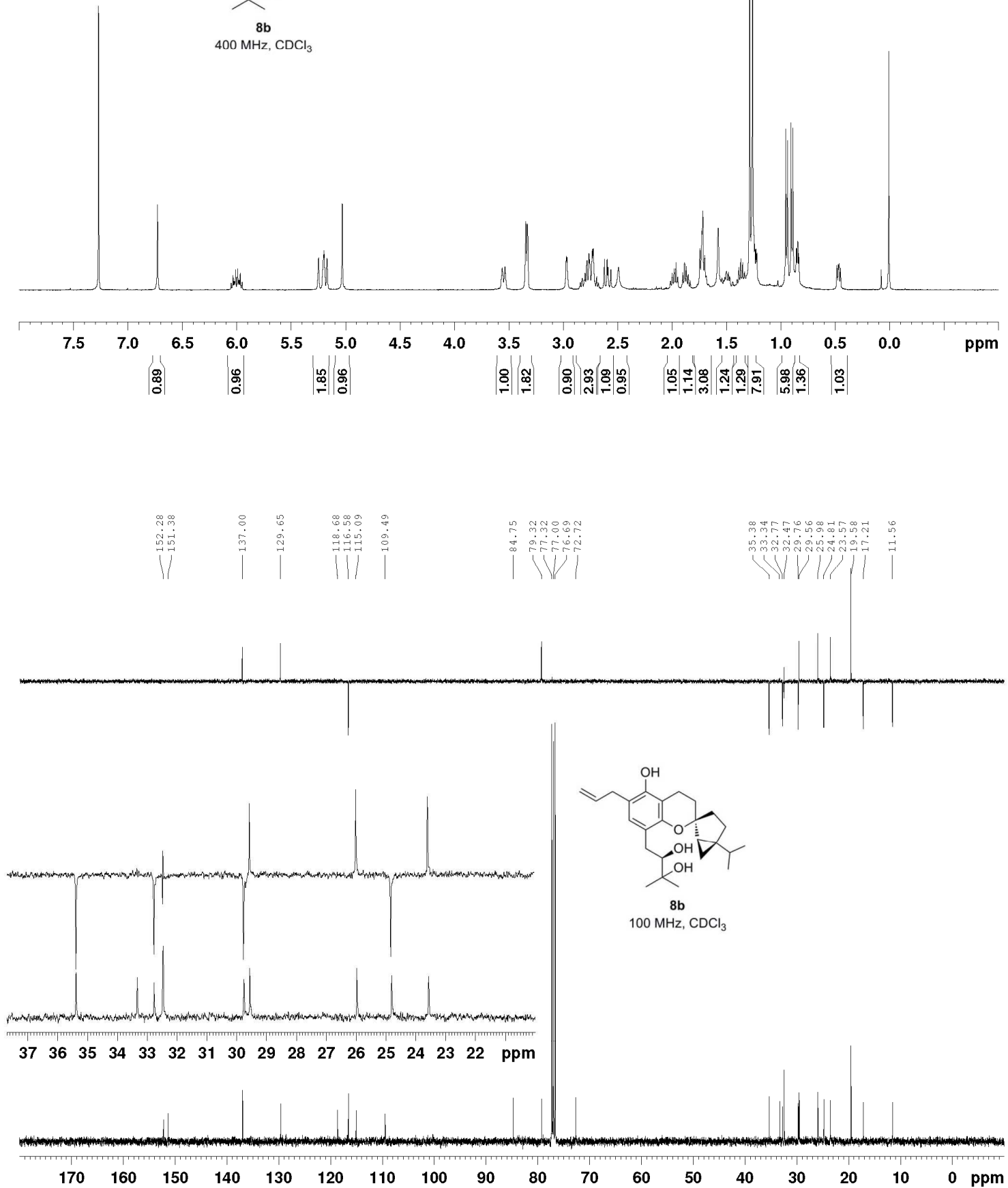


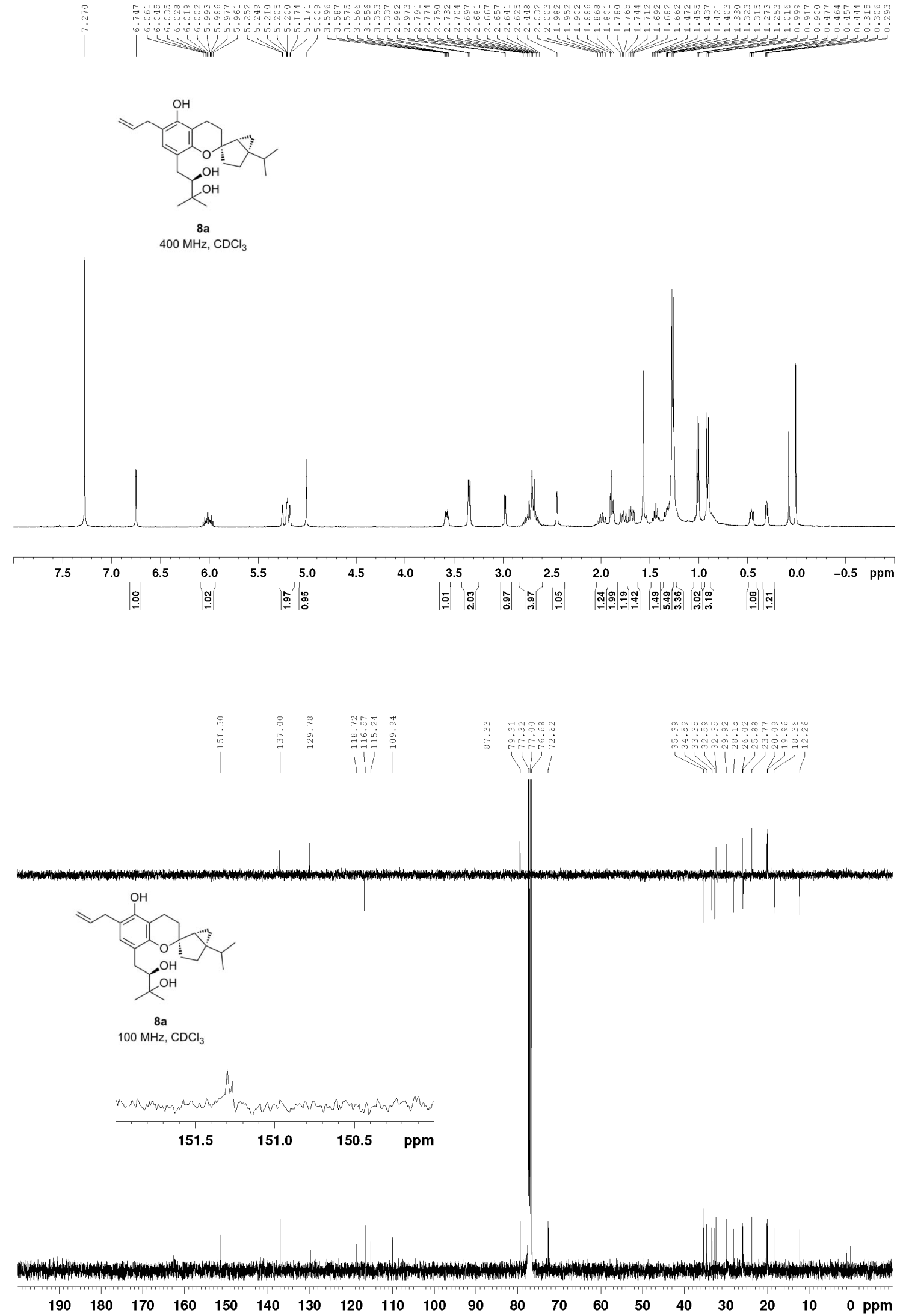




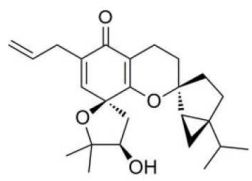

(-)-C4-epi-spirooliganone $\mathrm{B}$

$400 \mathrm{MHz}^{\mathrm{CDCl}} 3$

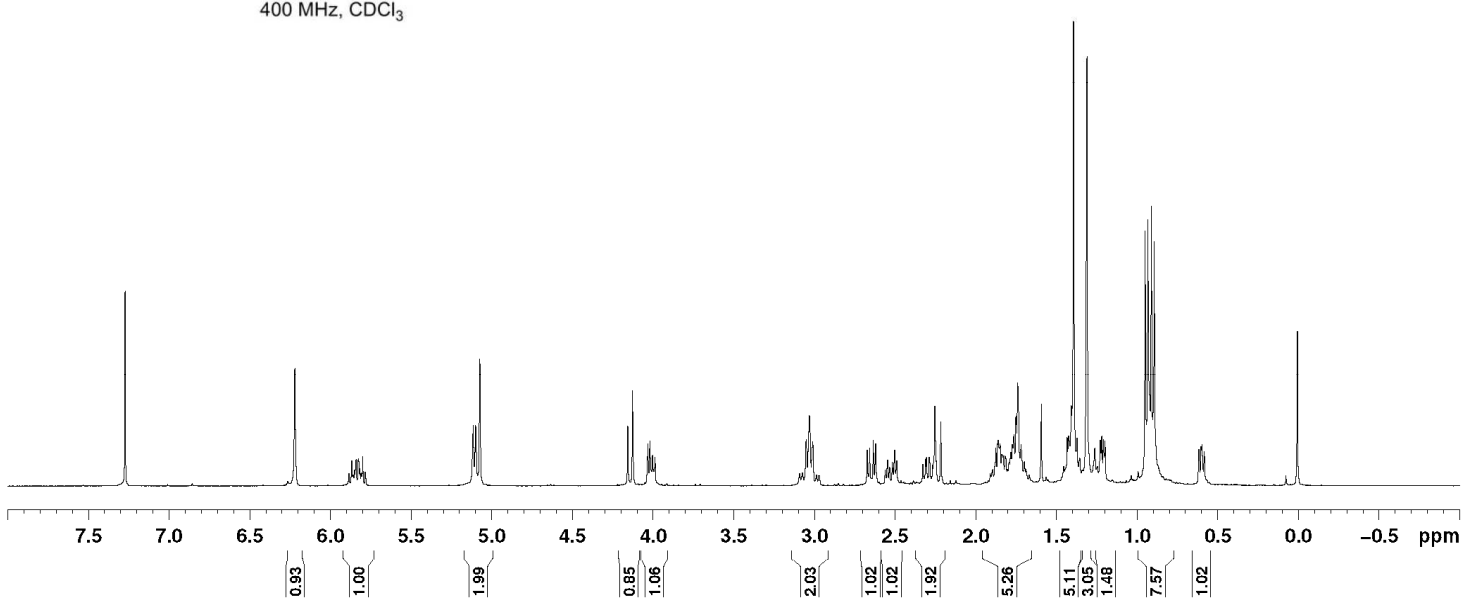

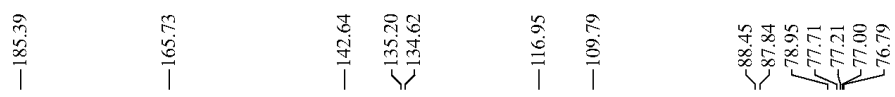
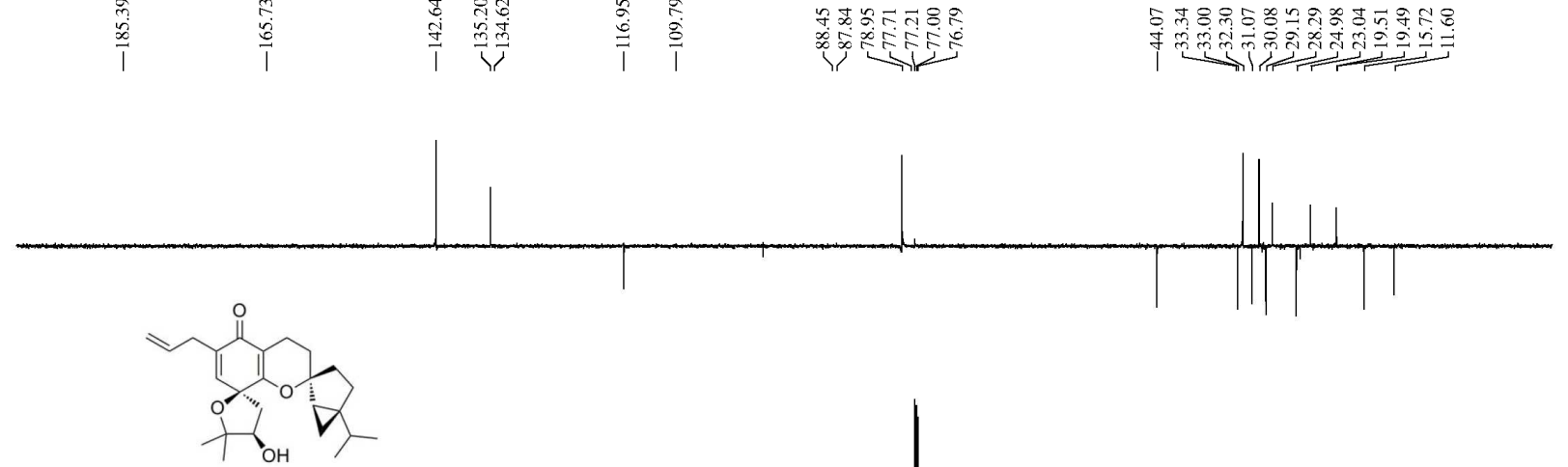

(-)-C4-epi-spirooliganone B

$150 \mathrm{MHz}, \mathrm{CDCl}_{3}$

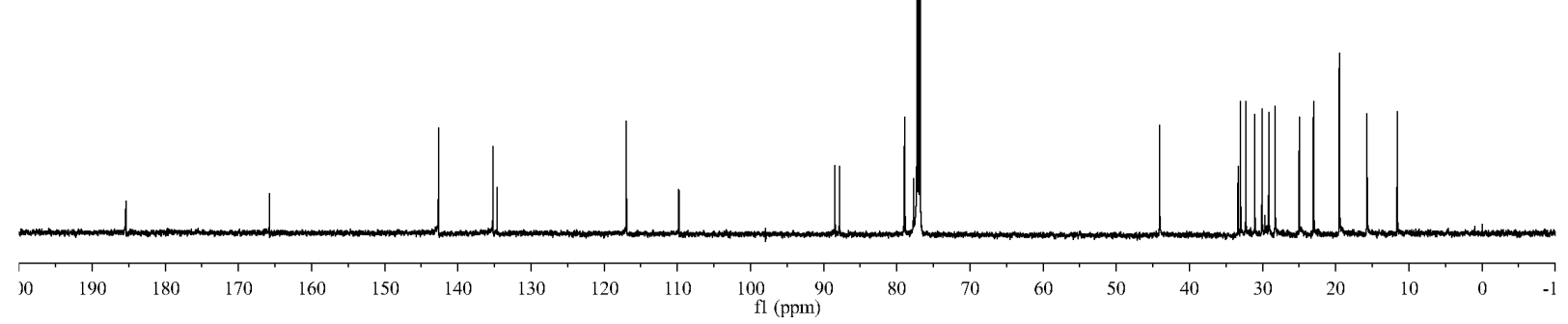



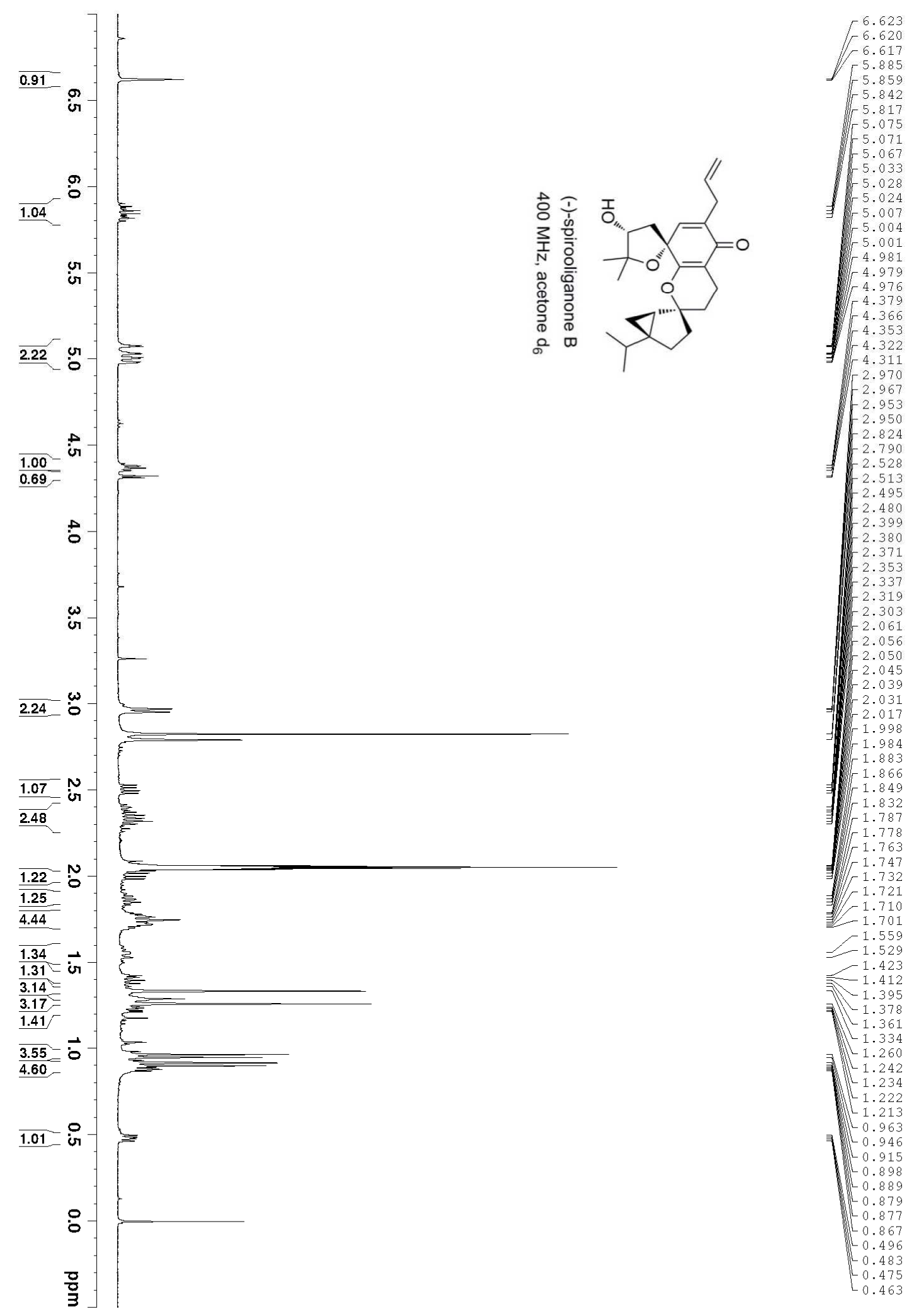

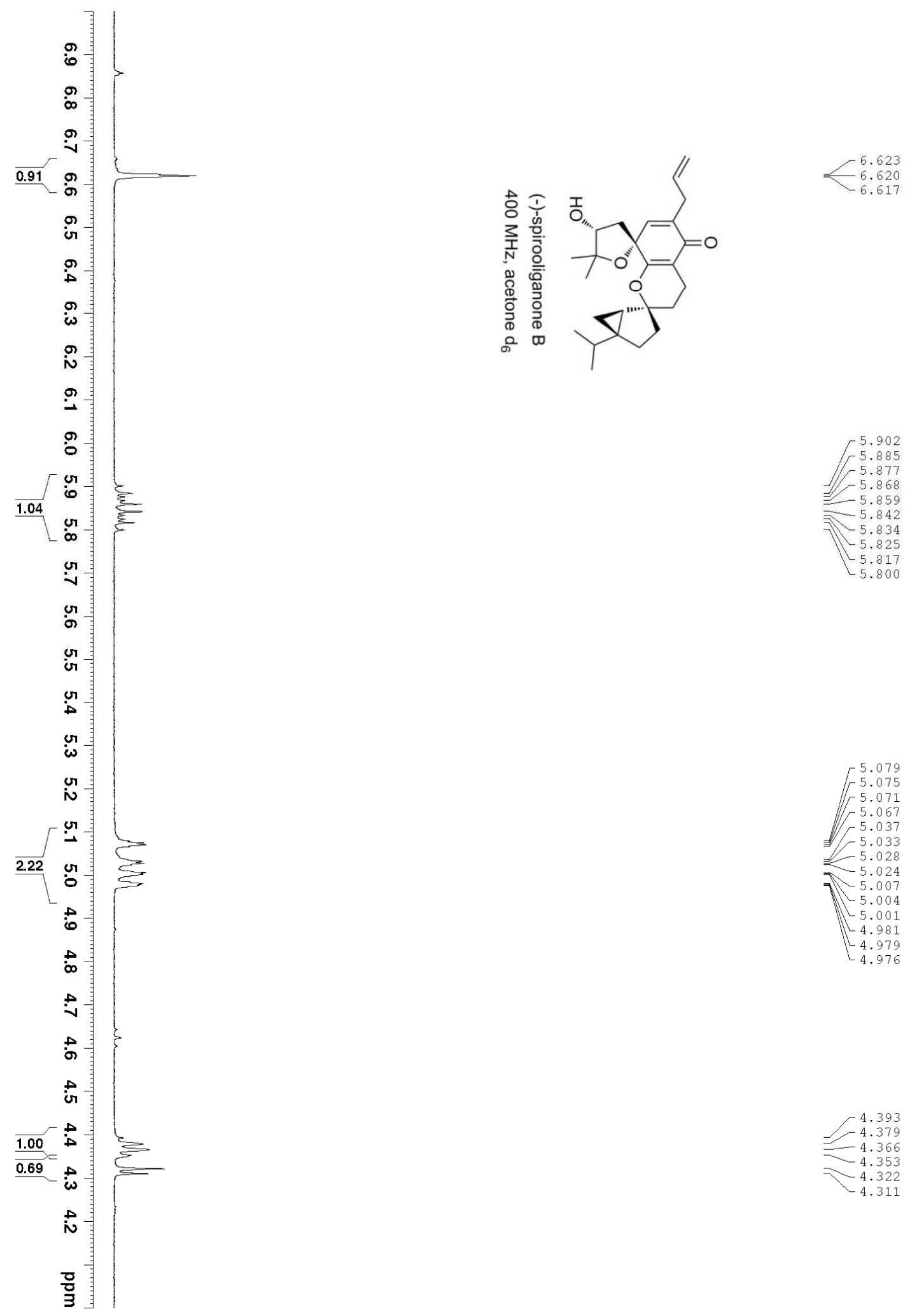

4.393

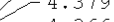

$-4.322$

$-4.311$ 

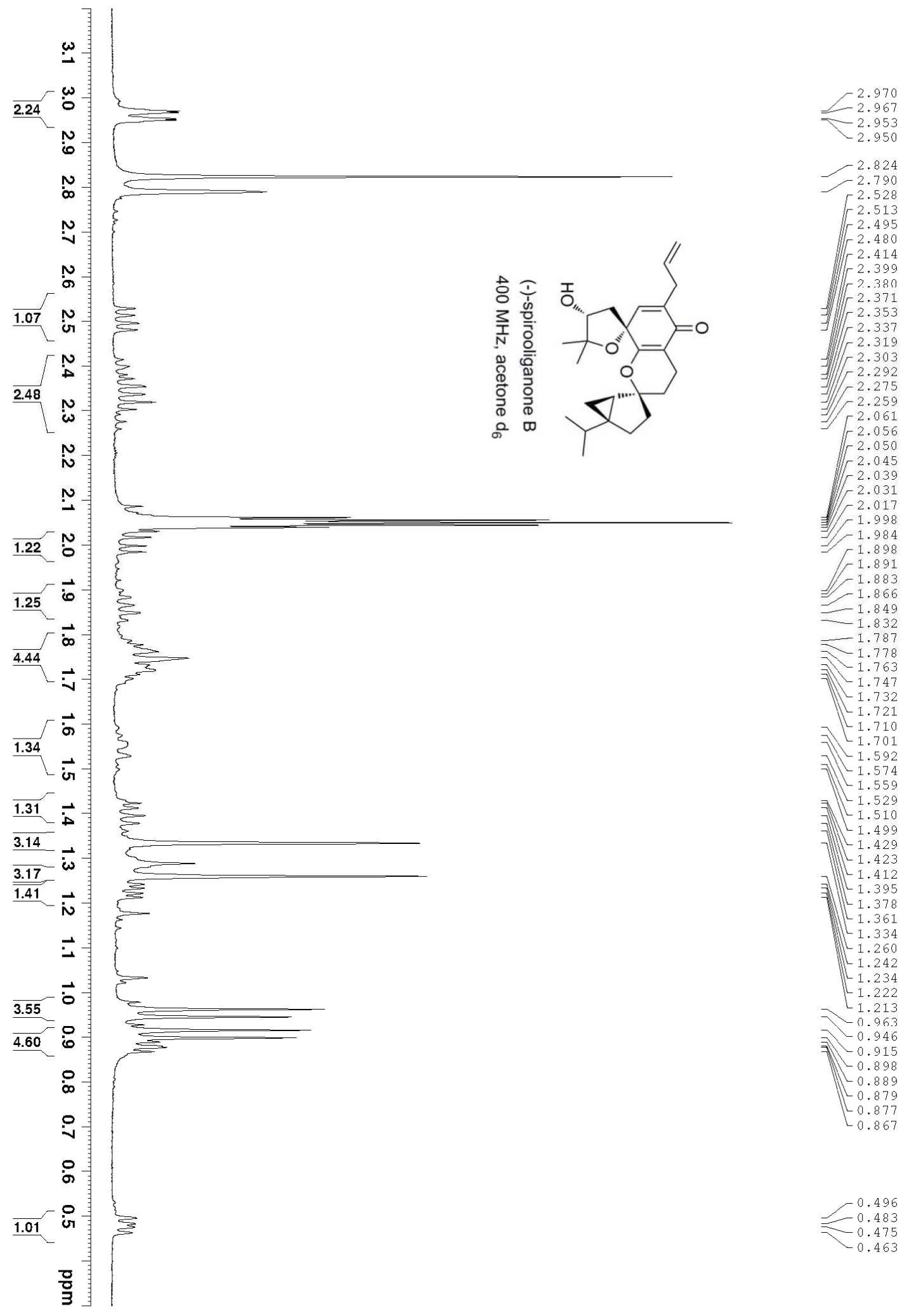


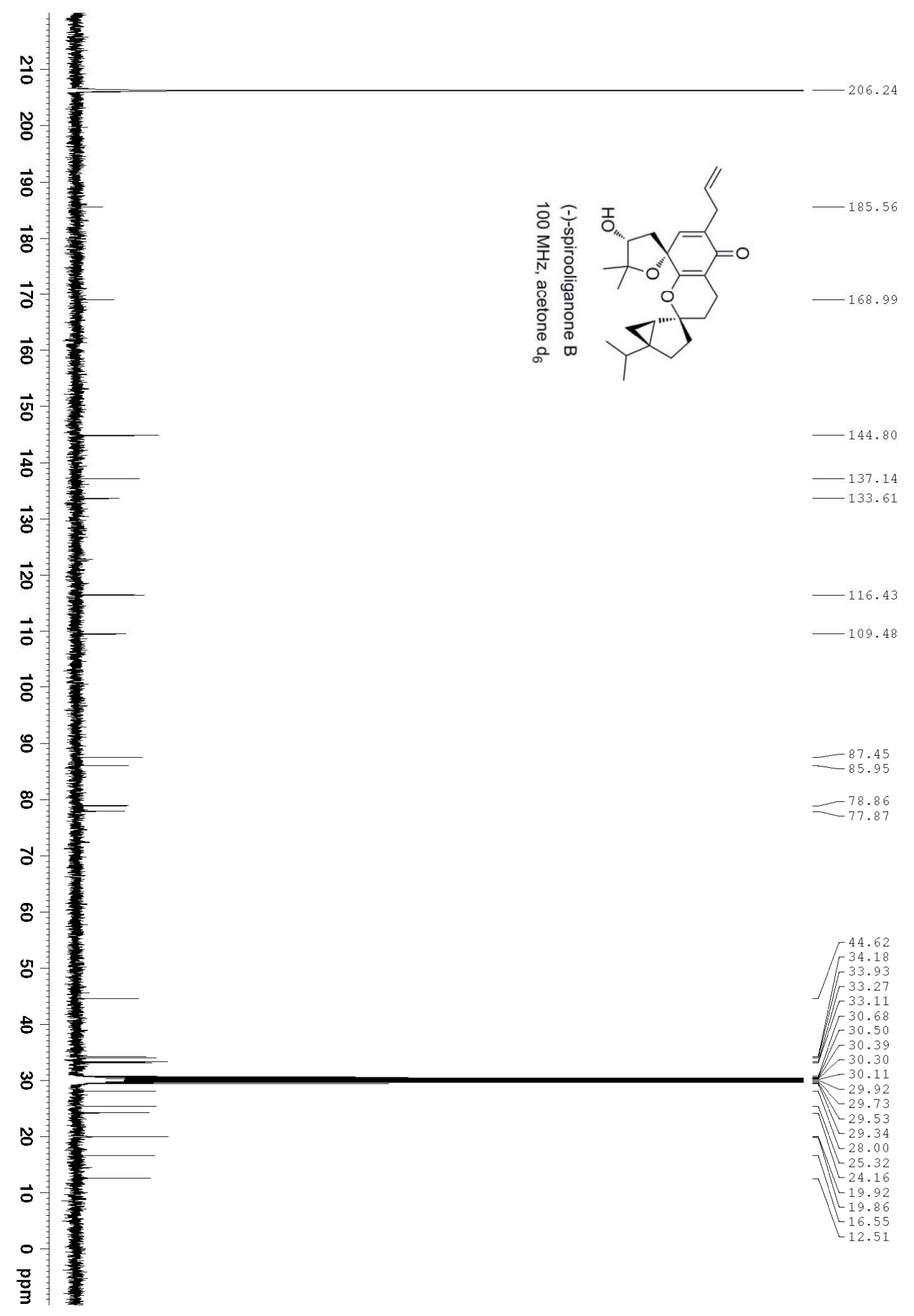




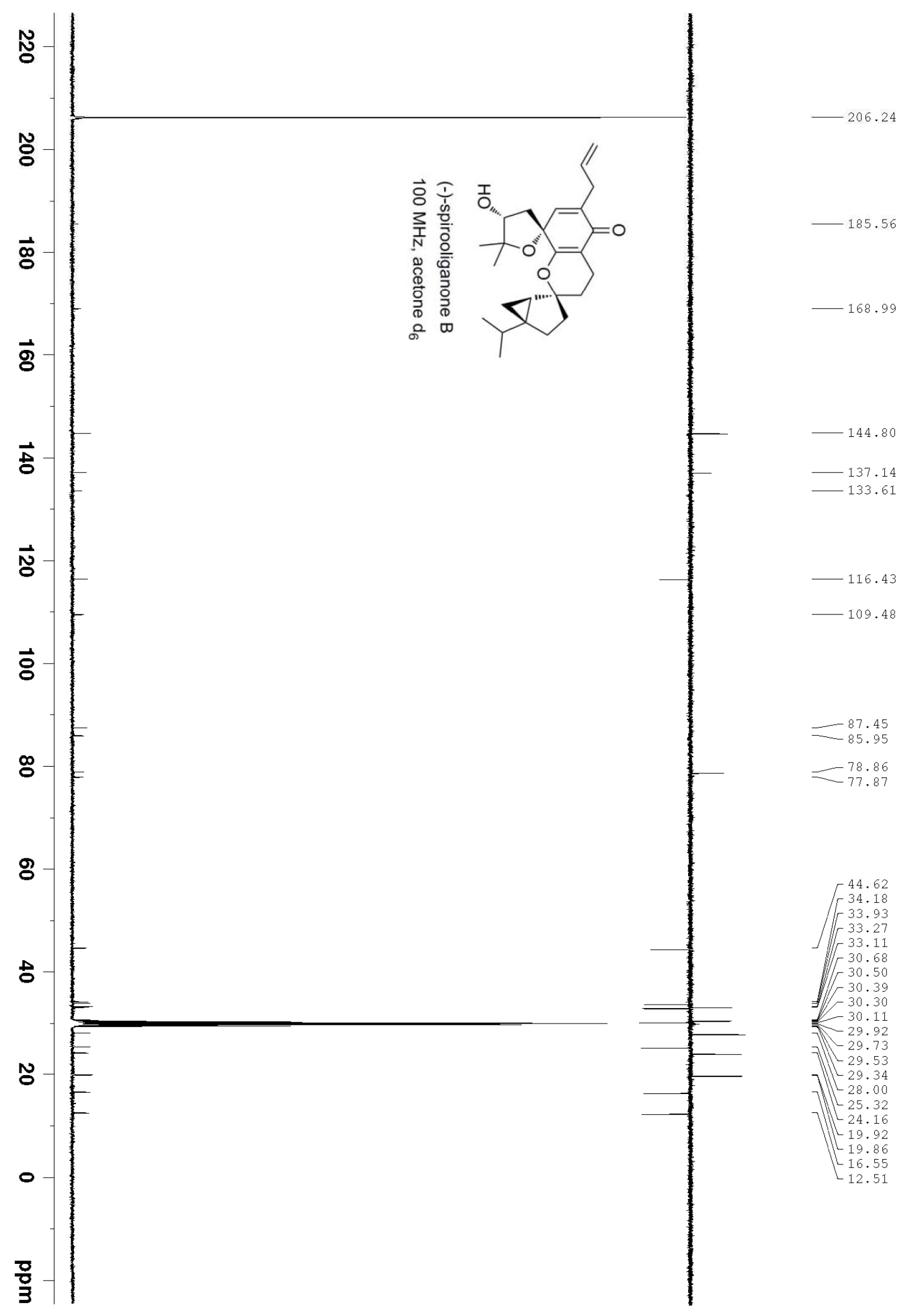



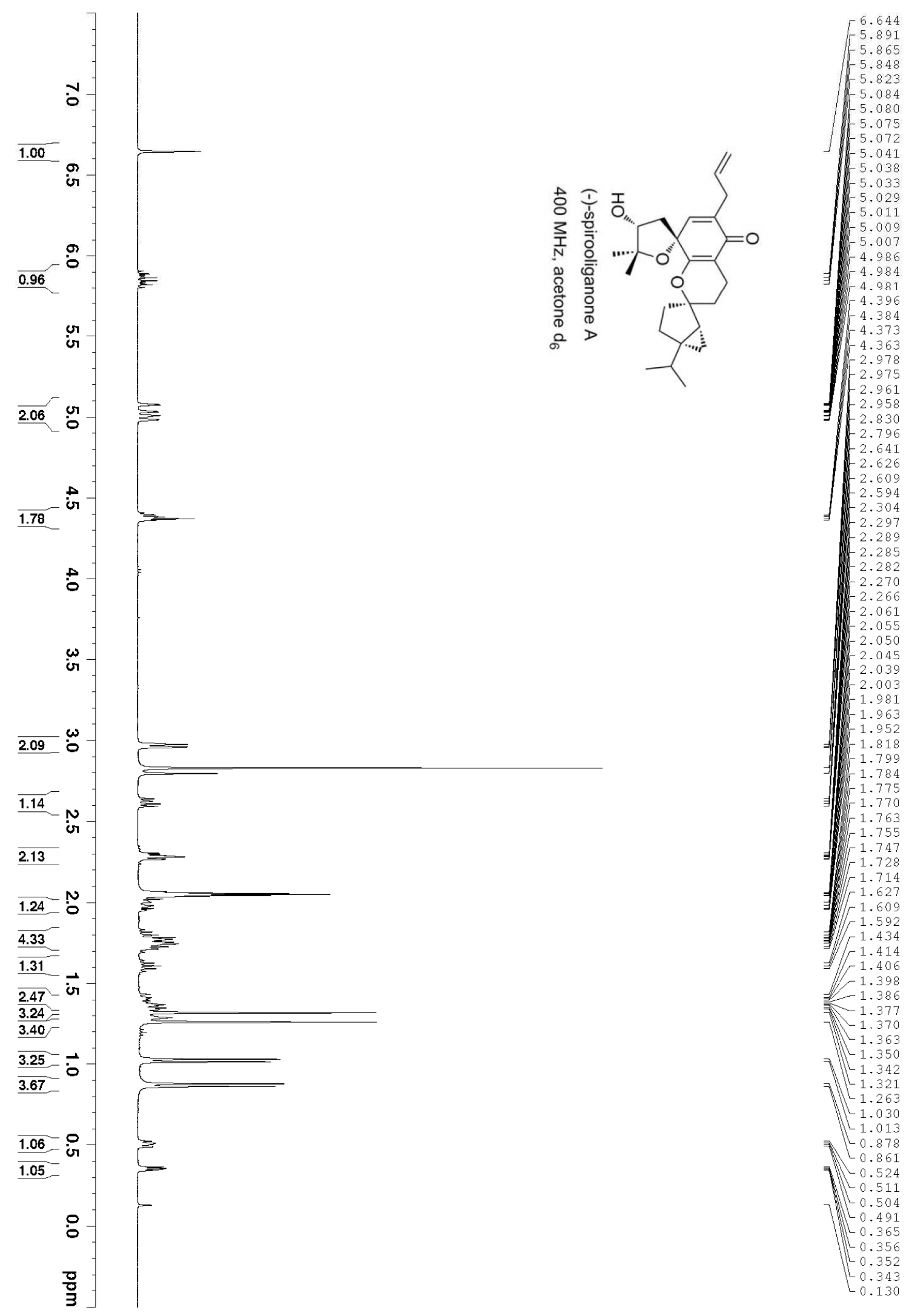

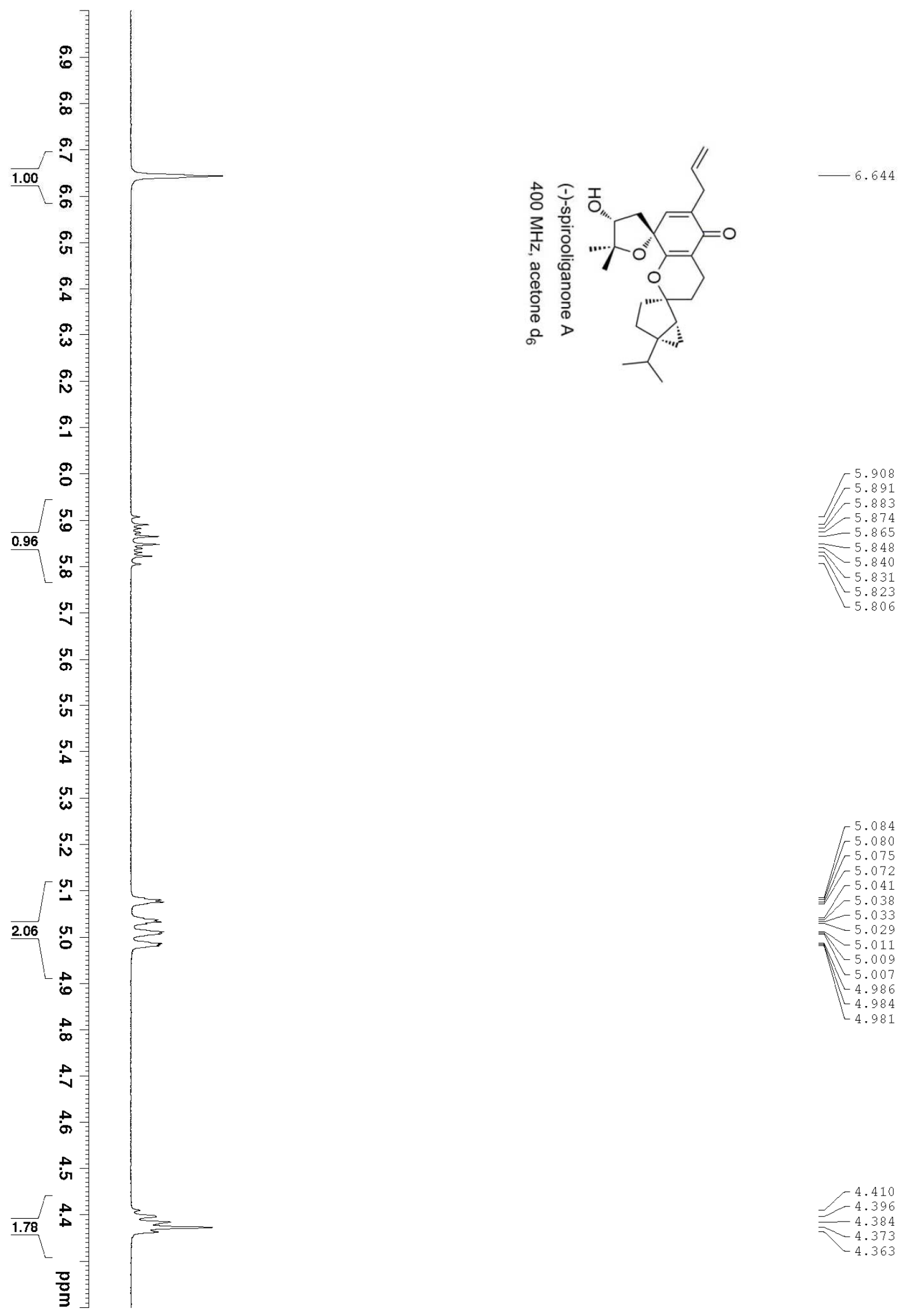


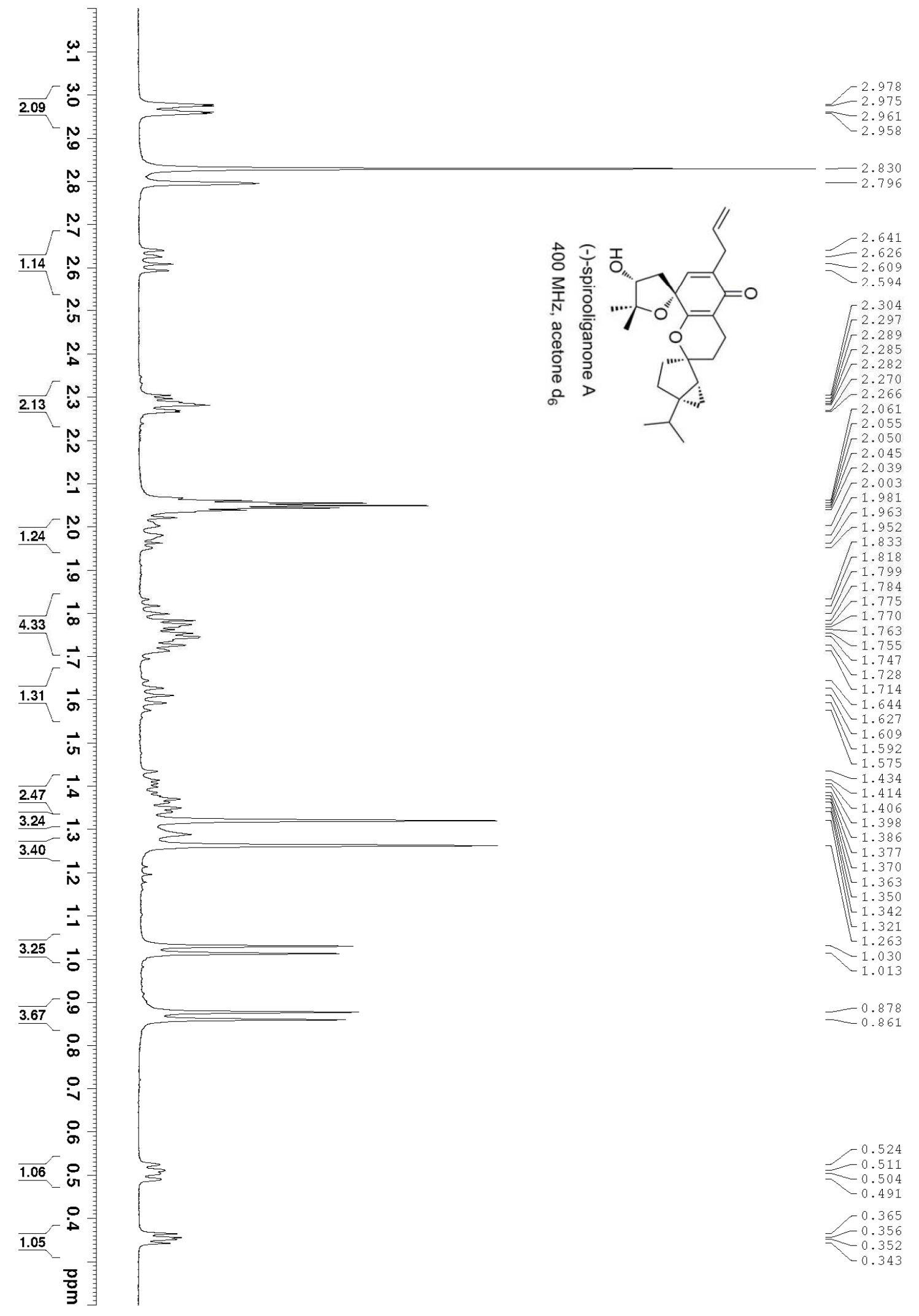




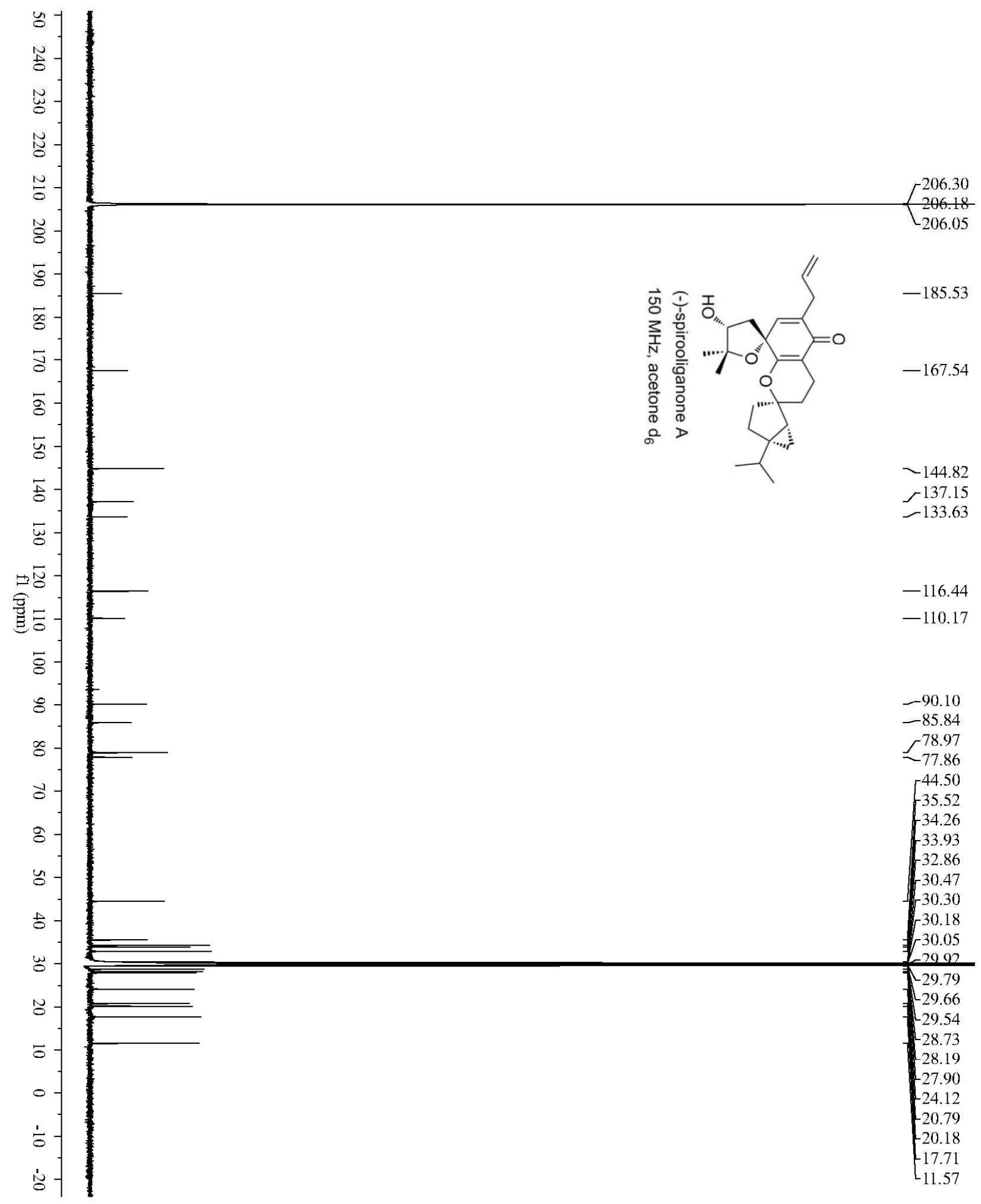




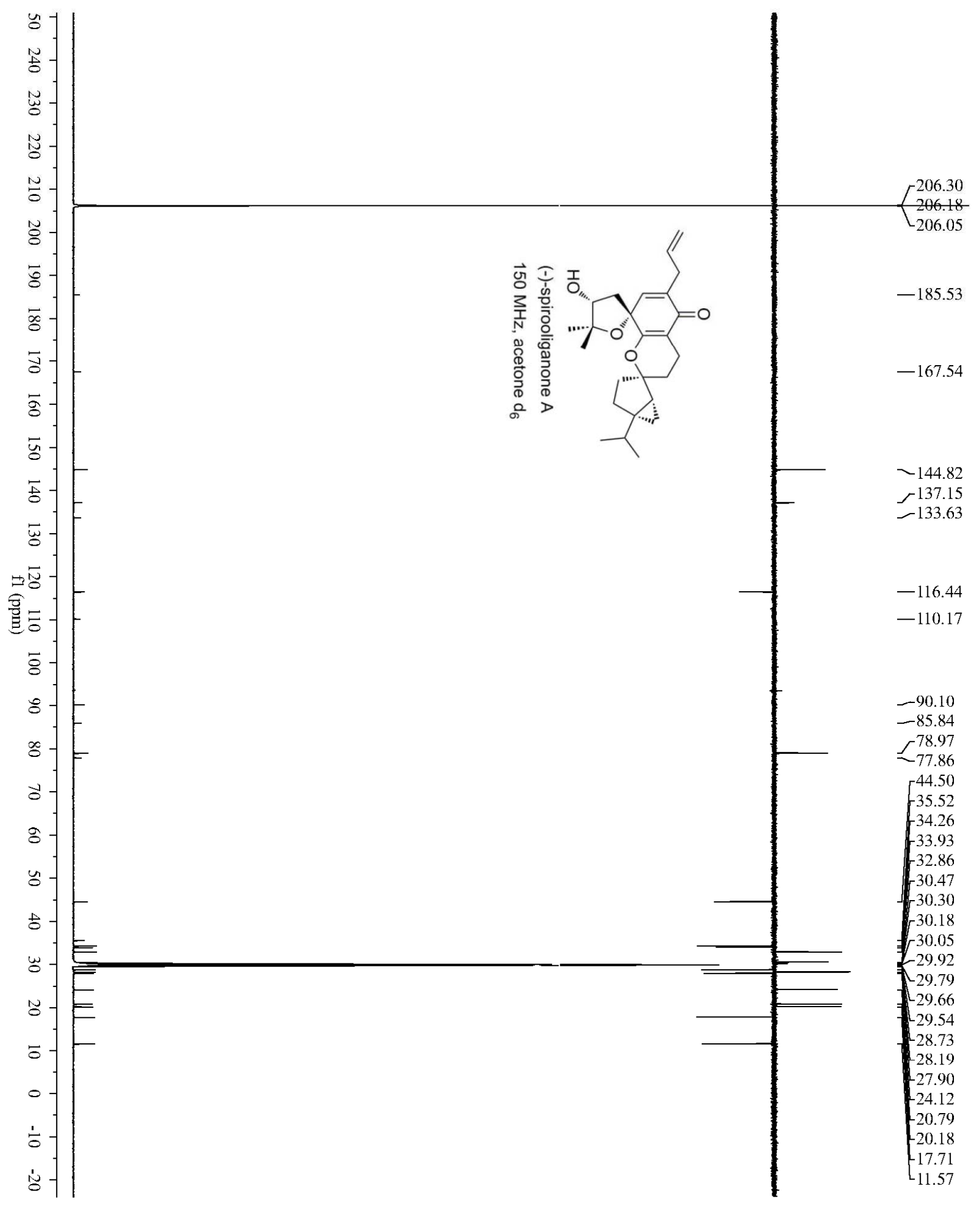

